\section{X-ray diagnostics of massive star winds}

\author{
Lidia M. Oskinova \\ Institute for Physics and Astronomy, University of \\ Potsdam, 14476 Potsdam, Germany
}

Abstract: Nearly all types of massive stars with radiatively driven stellar winds are X-ray sources that can be observed by the presently operating powerful X-ray telescopes. In this review I briefly address recent advances in our understanding of stellar winds obtained from X-ray observations. $\mathrm{X}$-rays may strongly influence the dynamics of weak winds of main sequence B-type stars. X-ray pulsations were detected in a $\beta$ Cep type variable giving evidence of tight photosphere-wind connections. The winds of OB dwarfs with subtypes later than O9V may be predominantly in a hot phase, and X-ray observations offer the best window for their studies. The X-ray properties of OB supergiants are largely determined by the effects of radiative transfer in their clumped stellar winds. The recently suggested method to directly measure mass-loss rates of $\mathrm{O}$ stars by fitting the shapes of X-ray emission lines is considered but its validity cannot be con-

Email address:

lida@astro.physik. uni-potsdam.de (Lidia M. Oskinova)

Preprint submitted to Advances in Space Research firmed. To obtain robust quantitative information on stellar wind parameters from $\mathrm{X}$-ray spectroscopy, a multiwavelength analysis by means of stellar atmosphere models is required. Independent groups are now performing such analyses with encouraging results. Joint analyses of optical, UV, and Xray spectra of OB supergiants yield consistent mass-loss rates. Depending on the adopted clumping parameters, the empirically derived mass-loss rates are comparable (within a factor of a few) to those predicted by standard recipes (Vink et al., 2001). All sufficiently studied O stars display variable X-ray emission that might be related to corotating interaction regions in their winds. In the latest stages of stellar evolution, single red supergiants (RSG) and luminous blue variable (LBV) stars do not emit observable amounts of X-rays. On the other hand, nearly all types of Wolf-Rayet (WR) stars are X-ray sources. $\mathrm{X}$-ray spectroscopy allows a sensitive probe of WR wind abundances and opacities.

\section{Introduction}

Stars much heavier than the Sun $\left(M_{\text {initial }}>8 M_{\odot}\right)$ are extremely luminous and drive strong stellar winds, blowing a large part of their matter into the galactic environment before they finally explode as a supernova. By this strong feedback, massive stars ionize, enrich, and heat the interstellar medium, regulate the star formation, and affect the further development of the cluster in which they were born. Empirical diagnostics of massive star spectra provide quantitative information about stellar and 
wind parameters, such as the terminal wind velocity, $v_{\infty}$, and the mass-loss rate, $\dot{M}$.

With the development of space based telescopes, the classical analyses of optical, and radio radiation was extended to the ultraviolet (UV) and the X-ray range. The optical range is the easiest to access. However in the vast majority of $\mathrm{OB}$ stars the optical spectra are dominated by photospheric lines. Only the most luminous stars with strongest stellar winds (such as Wolf-Rayet (WR) stars) show many wind lines in emission in the optical. OB stars commonly display wind signatures in their UV spectra, but UV observations are scarce. For main-sequence B stars the wind signatures are marginal and difficult to disentangle from the photospheric spectra even in the UV. In X-rays, on the other hand, we can observe emission lines from winds of nearly all types of massive stars, including Btype dwarfs and other stars with weak winds. Thus, X-rays provide an excellent, sometimes unique wind diagnostic.

The history of massive star X-ray astronomy begins with the UV observations. Back in the 1970's the observatory Copernicus made the important discovery of strong lines of highly ionized ions such as O vi, N v, and C IV in the UV spectra of massive stars as cool as spectral type B1. It became immediately clear that the stellar effective temperatures are not sufficiently high to power such high degrees of ionization. E.g. Hamann (1981) showed that the lines of O VI and N V observed in the UV spectra of the B-type star $\tau$ Sco cannot be reproduced by the standard wind models. While different theories were put forward to explain the presence of high ions in stellar spectra, it was the work of Cassinelli and Olson (1979) where the Auger ionization by X-rays was suggested as an explanation.

The first X-ray observations by the Einstein observatory indeed detected X-rays from OB stars, and hence proved the importance of the Auger process for stellar winds (Seward et al., 1979; Harnden et al., 1979). One of the instruments on board of the Einstein observatory was the Solid State Spectrometer (SSS) that was used to observe Otype stars.

Already at these early days of X-ray astronomy, Stewart and Fabian (1981) employed Einstein spectra to study the transfer of X-rays through a uniform stellar wind as a mean to determine stellar mass-loss rates. They applied a photoionization code to calculate the wind opacity. Using $\dot{M}$ as a model parameter, they found from matching the model and the observed X-ray spectrum of $\zeta$ Pup (O4I) that the X-ray based mass-loss rate is lower by a factor of a few than obtained from fitting the $\mathrm{H} \alpha$ emission line and the radio and IR excess. As the most plausible explanation for this discrepancy they suggested that the mass-loss rate derived from $\mathrm{H} \alpha$ is overestimated because of wind clumping. In retrospect, this was a deep insight confirmed by follow-up studies only in the 21st century.

These first low-resolution X-ray spectra of OB stars also showed emission signatures of such high ions as SXV and Si XIV, and thus revealed the presence of plasma with temperatures in excess of a few MK. From this moment on, a quest to explain X-rays from 
stellar winds has began. Among the first proposed explanations was a picture where the stellar wind has a very hot base zone where the plasma is constrained by a magnetic field. X-rays are produced in this base corona, and ionize the overlaying cool stellar wind. Models predict that X-rays originating from this inner part of the winds should become strongly absorbed in the overlaying outer wind. Therefore, when observations showed only little absorption of $\mathrm{X}$ rays, the base corona model was seriously questioned (Long and White, 1980; Cassinelli et al., 1981; Cassinelli and Swank, 1983). A way to resolve this "too little absorption" problem was shown only much later (see discussion in Section 8).

Besides the magnetically confined hot corona scenario, other models were put forward to explain X-ray emission from single massive stars. Detailed (albeit 1-D) radiative hydrodynamic models predict the development of strong shocks (Owocki et al., 1988; Feldmeier et al., 1997a; Runacres and Owocki, 2002) as a result of the line driving instability (LDI) intrinsic to radiatively driven stellar winds (Lucy and White, 1980; Lucy, 1982). A fraction of otherwise cool $\left(T_{\text {cool }} \sim 10 \mathrm{kK}\right)$ wind material is heated in these shocks to a few MK, and cools radiatively via X-ray emission. In the hydrodynamic model of Feldmeier et al. (1997b), the X-rays are generated when a fast parcel of gas rams into a slower-moving dense shell, both structures resulting from the LDI. This model was successful in quantitatively explaining the observed low-resolution Rosat spectrum of an $\mathrm{O}$ supergiant, and is often in- voked as the standard scenario for the origin of X-rays in stellar winds.

Another family of models suggests that radiatively driven blobs of matter plough through an ambient gas that is less radiatively accelerated (Lucy, 2012; Guo, 2010). These blobs might be the result of an instability (Lucy and White, 1980), or seeded at or below the stellar photosphere (Waldron and Cassinelli, 2009; Cantiello and Braithwaite, 2011). When these blobs propagate, forward shocks are formed, where gas is heated giving rise to X-ray emission. Cassinelli et al. (2008) \& Ignace et al. (2012) calculated the temperature and density in such a bow shocks to interpret some major X-ray properties: the power-law distribution of the observed emission measure derived from many hot star Xray spectra, and the wide range of ionization stages that appear to be present throughout the winds. One can also envisage a "hybrid" scenario to explain the X-ray emission from massive stars by a combination of magnetic mechanisms on the surface with shocks in the stellar wind (e.g. Cassinelli and Swank, 1983; Waldron and Cassinelli, 2009).

These and alternative models for the Xray production in stellar winds are now rigorously checked by modern observations. The 21st century's X-ray telescopes Chandra and XMM-Newton made high-resolution X-ray spectroscopy possible (Brinkman et al., 2000; den Herder et al., 2001; Canizares et al., 2005). On board of Chandra is the High Energy Transmission Grating Spectrometer (HETGS/MEG) with a spectral resolution of $0.024 \AA$ in its 1st order. The Reflection Grating Spectrometers (RGSs) of XMM-Newton 


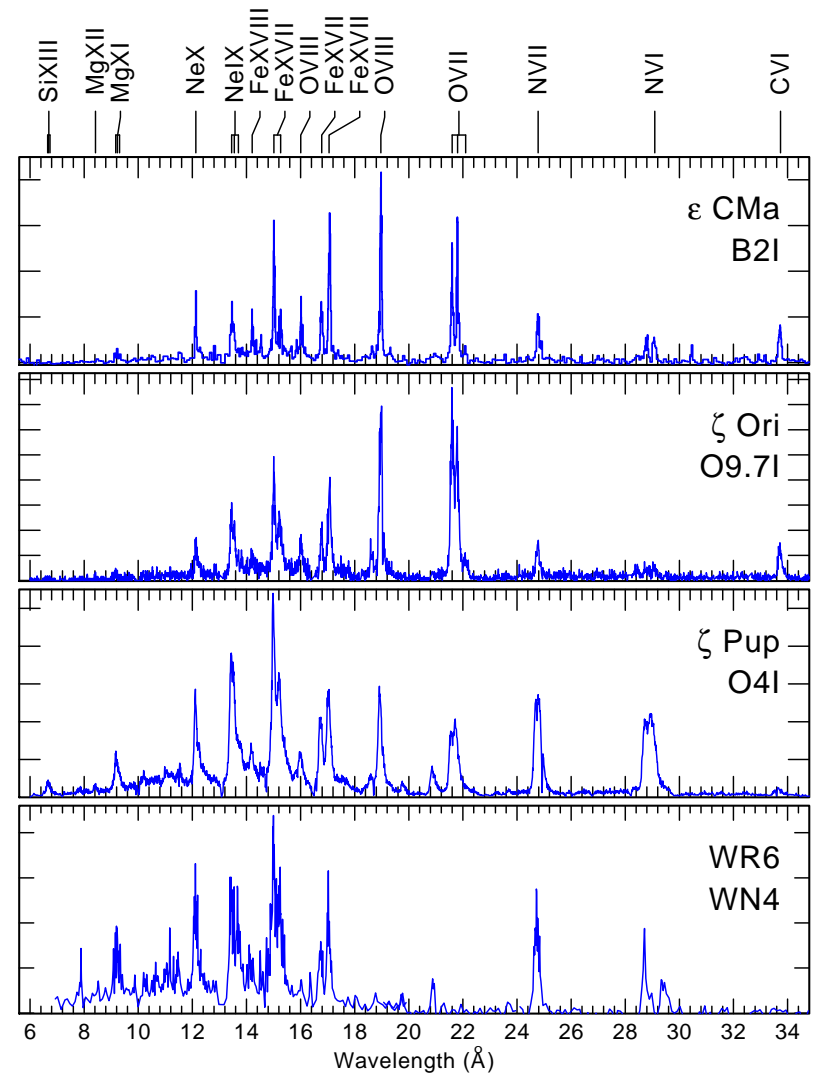

Figure 1: A sample of high-resolution X-ray spectra obtained with the RGS spectrograph on board of $X M M-N e w t o n$. The vertical axis is the $\mathrm{X}$-ray count rate in arbitrary units. Strong lines are identified at the top.

have a more modest spectral resolution of $0.05 \AA$, but higher sensitivity. Figure 1 shows examples of high-resolution X-ray spectra. Both telescopes also provide a possibility for low-resolution X-ray spectroscopy, imaging, and timing analysis.

In this review I briefly address the diagnostic potential of X-rays for our understanding of winds from single stars. General X-ray properties of massive stars are introduced in
Section 2, and the location of X-ray plasma in their winds is discussed in Section 3. The classical UV diagnostics of stellar winds and the influence of $\mathrm{X}$-rays on these diagnostics are considered in Section 4. The role of Xrays in resolving the so-called "weak wind problem" is discussed in Section 5. The Xray properties of $\mathrm{B}$-stars, including pulsating $\beta$ Cep-type variables and magnetic stars, are briefly considered in Section 6. Section 7 deals with using X-ray variability to study the structure of stellar winds. Section 9 introduces modeling approaches for the description of X-ray line spectra. The diagnostic power of X-ray emission lines is discussed in Section 8. Modern approaches combining X-ray spectroscopy with a multiwavelength analysis are presented in Section 10. Xray diagnostics of massive stars in the latest stages of their evolution, RSG, WR, and LBV stars, are considered in Section 11. A summary concludes this review (Section 12).

\section{General X-ray properties of massive stars}

Already in the dawn of stellar X-ray astronomy it was noticed that the X-ray luminosities of $\mathrm{O}$ stars roughly correlate with their bolometric luminosities as $L_{\mathrm{X}} \sim 10^{-7} L_{\text {bol }}$ (Long and White, 1980; Cassinelli et al., 1981; Pallavicini et al., 1981; Berghoefer et al., 1997). Follow-up investigations largely confirmed this empirical correlation and established that it holds not only for single $\mathrm{O}$ stars but also for binaries (Oskinova, 2005; Nazé, 2009).

The $L_{\mathrm{X}} \sim 10^{-7} L_{\mathrm{bol}}$ correlation holds over 
a limited range of spectral types, and apparently does not extend to stars with weaker nor with stronger winds than O-type stars. E.g. in their studies of the Orion Nebula Cluster (ONC), Stelzer et al. (2005) showed that the X-ray properties of late $\mathrm{O}$ and early $\mathrm{B}$ dwarfs are diverse, with luminosities that differ by orders of magnitude from the correlation $L_{\mathrm{X}} \sim 10^{-7} L_{\mathrm{bol}}$. On the basis of their studies of the star cluster NGC6231, Sana et al. (2006) noticed that the X-ray emission from B stars cannot be considered as a continuation of the $\mathrm{O}$ star X-ray properties to lower luminosities. Hardly any correlation between $L_{\mathrm{X}}$ and $L_{\mathrm{bol}}$ was found for B stars in the Carina Nebula (Nazé et al., 2011). The X-ray luminosity steeply drops at spectral types later than B2 (Cassinelli et al., 1994). Interestingly, this decline occurs roughly at the minimum bolometric luminosity required to initiate and sustain a radiatively driven stellar wind (Abbott, 1979). On the other hand, stars with strong winds, such as single WR stars, also do not follow an $L_{\mathrm{X}} \propto L_{\text {bol }}$ correlation (Wessolowski, 1996; Ignace and Oskinova, 1999; Ignace et al., 2000; Oskinova, 2005).

The physics behind $L_{\mathrm{X}} \propto L_{\mathrm{bol}}$ is not yet understood. Chlebowski and Garmany (1991) found a dependence between the wind energy of the single stars and their X-ray luminosity, and suggested that this dependence is at least partially responsible for the proportionality between $L_{\mathrm{X}}$ and $L_{\mathrm{bol}}$. Owocki and Cohen (1999) and Ignace and Oskinova (1999) suggested that a specific radial distribution of the X-ray filling factor may explain the $L_{\mathrm{X}} \propto L_{\mathrm{bol}}$ correlation for OB stars, and the lack of such correlation for WR stars. Oskinova et al. (2011a) considered the possibility that the X-ray luminosity is correlated with the stellar parameters via a dependence of the properties of the subphotospheric convective zone on $L_{\mathrm{bol}}$ and $T_{\mathrm{eff}}$. E.g., Cantiello and Braithwaite (2011) predict that surface magnetic fields are common in OB stars, and their strength is increasing for hotter and more luminous stars. The wind momentum is also increasing with luminosity (Castor et al., 1975; Puls et al., 2006). Thus, the ratio of the wind kinetic energy to the magnetic energy could remain similar for stars of different luminosities. Interestingly, Walborn et al. (2009) found from a study of high-resolution X-ray spectra of O star that the X-ray plasma temperature correlates with the stellar effective temperature, indicating a close association between the hot plasma and the stellar (sub)photosphere.

On the other hand, Owocki et al. (2013) further investigated the idea that the $L_{\mathrm{X}} \propto$ $L_{\text {bol }}$ correlation is secondary to a correlations between $L_{\mathrm{X}}$ and stellar wind properties. In their study of radiative shocks, it was found that accounting for the thin-shell instability (Vishniac, 1994) leads to a correlation $L_{\mathrm{X}} \propto\left(\dot{M} / v_{\infty}\right)^{1-m}$, where $m$ is some exponent. Making the assumption $m \approx 0.4$ results in the observed linear $L_{\mathrm{X}} \propto L_{\mathrm{bol}}$ correlation. This model also provides a natural explanation why stars with thin winds, such as B dwarfs, and stars with thick winds, such as WR stars, have a different scaling between $\mathrm{X}$-ray and bolometric luminosity than the Otype stars (see review by K. Gayley in this volume). Future work should show how real- 
istic is the mixing exponent ansatz.

The X-ray spectra of single massive stars are, in general, well described by optically thin multi-temperature plasma in collisional equilibrium. The spectra are comparably soft - the emission measure weighted mean temperature usually does not exceed a few MK (e.g. Pallavicini, 1989; Zhekov and Palla, 2007; Nazé, 2009). The X-ray emission is remarkably constant on short time scales of a few hours, but typically shows non-coherent slow fluctuations on a few percent level and on the time scale of days. While the former time scale (hours) is comparable to the dynamical time of the stellar wind, the latter time scale (days) is likely connected with stellar rotation.

To summarize, a normal massive OB star is a nearly constant (not flaring) X-ray source with a luminosity $\sim 10^{-7} L_{\text {bol }}$ and a thermal $\mathrm{X}$-ray spectrum with an average temperature of a few MK.

\section{Location of the hot X-ray emitting plasma in $\mathrm{OB}$ star winds}

High-resolution spectroscopy allows to constrain the location of hot emitting plasma. This can be done by analyzing spectra of Helike ions. which display prominent lines in the X-ray spectra of massive stars (see Fig. 1). The spectrum of a He-like ion consists of a resonance line, $r$, intercombination lines $i$, and a highly forbidden line $f$. The ratios between the fluxes in these lines are strongly sensitive to the conditions in the emitting plasma. For instance, the ratio of fluxes between the forbidden and intercombination

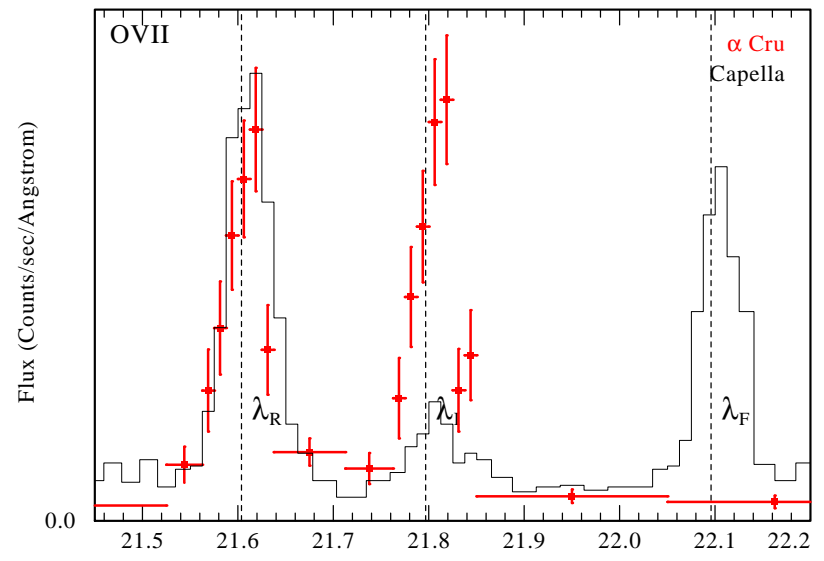

Figure 2: The O vil fir triplet $\left(\lambda_{\mathrm{w}}=21.60 \AA, \lambda_{\mathrm{xy}}=\right.$ $21.8 \AA, \lambda_{\mathrm{z}}=22.098 \AA$ ) in the Chandra LETGS spectra of $\alpha$ Cru (B0.5IV) (red symbols) and Capella (G5III) (black histogram). The central wavelengths of the $r, i$, and $f$ lines are indicated by dashed vertical lines. The $r$ lines from both stars are normalized to the same height. In the spectrum of $\alpha \mathrm{Cru}$ the forbidden line is strongly suppressed due to the effect of strong stellar UV radiation. Based on our Chandra observations of $\alpha \mathrm{Cru}$ and the archival Chandra data of Capella.

components, $\mathcal{R}$, is sensitive to the electron density and the ultraviolet flux (Blumenthal et al., 1972; Porquet et al., 2001). The UV radiation suppresses the forbidden line and enhances of the intercombination line, mimicking the effect of higher electron density. In typical OB-type stars, their copious UV radiation is the dominant cause for forbidden line depopulation (Fig. 2).

If the stellar UV photospheric flux, $H_{\nu}$, is known, the geometrical dilution factor $W$ can be inferred from measured value $\mathcal{R}\left(W H_{\nu}\right)$. This constrains the radius $R_{\text {fir }}$ where the Xray emitting plasma is located (e.g. Waldron and Cassinelli, 2001; Leutenegger et al., 2006; 
Shenar et al., 2015).

One of the first surprises brought by the Chandra and XMM-Newton spectroscopy of OB stars was the discovery that the hot plasma is located not far out in the wind, but instead resides already very close to the stellar photosphere (Waldron and Cassinelli, 2001; Kahn et al., 2001).

Furthermore, Waldron and Cassinelli (2007) pointed out a "high-ion near star problem": lines of ions with higher ionization potential are formed closer to the stellar surface than those of lower ions. Particularly, the line formation regions of Si XIII and S XV in the Osupergiant $\zeta$ Ori (O9.7Ib) are very close to the stellar photosphere, where X-rays emission is not expected theoretically (Feldmeier et al., 1997b; Krtička et al., 2009). The proposed explanation invoked surface magnetic fields. Soon after, a surface magnetic field was indeed detected on $\zeta$ Ori (Bouret et al., 2008). Interestingly, Berghofer and Schmitt (1994) reported an X-ray flare from this star. The recent development in stellar structure modeling shows that small-scale surface magnetic fields may be common in massive stars (e.g. Cantiello and Braithwaite, 2011).

From the analysis of a sizable sample of OB star X-ray spectra, Waldron and Cassinelli (2007) found a good correlation between the $f / i$-inferred radii and the radii where the X-ray continuum optical depth is unity. This provides evidence for the absorption of X-rays in the stellar wind, in agreement with theoretical expectation.

Recently, large progress was made in improving the $f / i$ line ratio diagnostics by providing a more realistic description of the UV radiation field in the stellar wind. For wavelengths in the observable part of the UV spectrum, the flux $H_{\nu}$ can be, in principle, directly inferred from observations. Unfortunately, especially for giant and supergiant stars, the photospheric fluxes are often contaminated by wind lines. E.g. the O vi resonance doublet overlaps with the $\lambda_{\mathrm{f} \rightarrow \mathrm{i}}$ transition at $1033 \AA$ for Mg XI. The O vi doublet itself can only be reproduced by stellar atmosphere models if the X-ray field causing Auger ionization is included (Oskinova et al., 2006). This example highlights the need of a consistent radiative transfer treatment for the correct interpretation of the fir line ratios. E.g. Oskinova et al. (2012), Hervé et al. (2012), Shenar et al. (2015), Rauw et al. (2015b), Puebla et al. (2016) independently used sophisticated NLTE stellar atmosphere codes to predict the $f / i$ line ratio in dependence on radius. Empirical $f / i$ line ratios can be measured from the observed high-resolution $\mathrm{X}$-ray spectra of OB stars and compared to the model predictions, allowing to constrain the plasma location. Comparison between the model and observations show that X-ray emission in OB supergiants has onset radius below $\sim 1.5 R_{*}$ and is distributed over a large range of radii. These findings strongly constrain the models of X-ray production in massive stars.

\section{Influence of X-rays on stellar wind ionization balance: including $\mathrm{X}$-rays in the UV wind diagnostics}

The usual empirical diagnostics of winds from OB stars is by analysis of UV resonance 
lines of abundant metal ions. By comparing observed and model lines of an ion in some ionization stage, the product of mass-loss rate and the ionization fraction is derived. As a next step, an assumption about the ionization fraction is made, e.g. it may be assumed that the given ionization stage is the leading one. Based on this assumption, the mass-loss rate can be evaluated.

A puzzling result was obtained when Prinja (1989) applied this method, to the UV high-resolution spectra of 40 non-supergiant $\mathrm{B}$ stars obtained with the IUE observatory. It was found that, in general, wind velocities are in the range of a few $\times 100 \mathrm{~km} \mathrm{~s}^{-1}$ and do not exceed the photospheric escape velocity. It was also found that the wind column densities are not significant - the product of mass-loss rates and ionization fractions of leading ions are small, e.g. $\log (\dot{M} q(\mathrm{C}$ III $))<$ $-10\left[M_{\odot} \mathrm{yr}^{-1}\right]$.

However, the presence of X-rays changes the wind ionization balance and, hence, may strongly affect the UV wind diagnostics (e.g. Waldron, 1984; Macfarlane et al., 1994; Massa et al., 2003, see also the review by Krticka \& Kubat in this volume). Waldron and Cassinelli (2010) pointed out that if X-rays are not included in the models, the ionic fractions could be incorrectly estimated leading to errors in the derived mass-loss rate. Figure 3 demonstrates the influence of X-rays on the empirical mass-loss diagnostics based on the modeling of UV resonance lines. In $\tau$ Sco, the observed C IV doublet can be reproduced without X-ray superionization only by assuming a very low mass-loss rate. On the other hand, including X-rays at the ob- served level in the models improves the fits and allows to reproduce the C IV doublet with a higher mass-loss rate. This example shows that X-rays should be included in the stellar atmosphere models to obtain correct empirical mass-loss rates from the analysis of UV spectra.

Such analysis was done by Oskinova et al. (2011b), who included the X-ray field in the detailed modeling of the UV spectra of a sample of non-supergiant B stars. It was shown that the models are in agreement with empirical results of Prinja (1989) - the mass-loss rates in main-sequence $\mathrm{B}$ stars are low, with $\log \dot{M}<9$, and the wind velocities are of the order of the wind escape velocity.

The influence of X-rays on the ionization structure of stellar winds was also considered as a possible solution for the very low massloss rates derived from modeling UV resonance lines, such as e.g. $\mathrm{P}_{\mathrm{V}} \lambda \lambda 1118,1128 \AA$ resonance doublet (Massa et al., 2003; Bouret et al., 2005; Marcolino et al., 2009). This line is usually unsaturated and, in principle, can serve as a robust mass-loss indicator.

Bouret et al. (2005) and Fullerton et al. (2006) found that mass-loss rates obtained from modeling $\mathrm{P}$ V in O-star spectra are significantly lower than those measured from the emission $\mathrm{H} \alpha$ line (see Section 8.1). Waldron and Cassinelli (2010) suggested that strong emission line radiation in the XUV band (between 54 to $124 \mathrm{eV}$ ) near the $\mathrm{P}$ V ionization edge can significantly change the ionization structure of the wind. This could significantly reduce the fractional abundance of $\mathrm{P} V$ and hence skew the UV-line diagnostics based on the assumption that $\mathrm{PV}_{\mathrm{V}}$ is the leading 


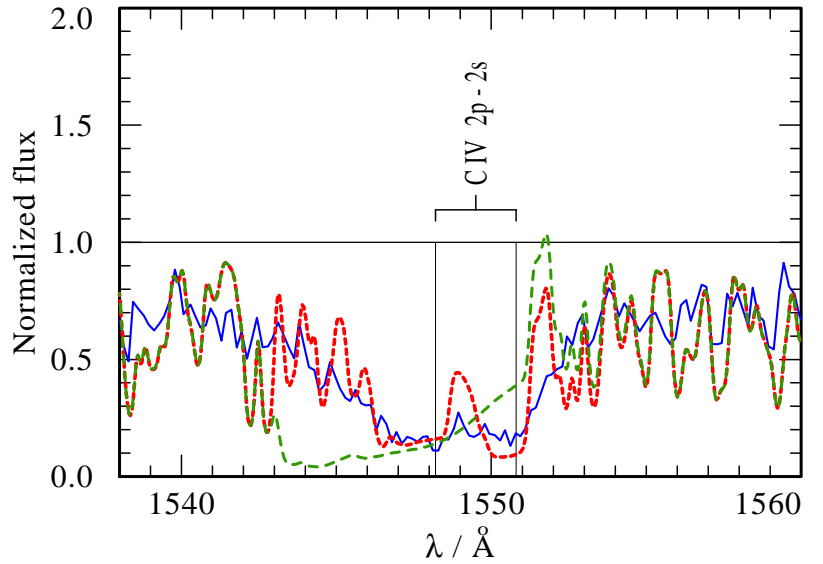

Figure 3: The effect of ionization by X-rays on the C IV $\lambda \lambda 1548.2,1550.8 \AA$ doublet. Detail of the UV spectrum of $\tau$ Sco $(\mathrm{B} 0.2 \mathrm{~V})$ as observed with IUE (blue thin line) vs. PoWR models: without X-rays (green line) and with X-rays (red line). The model parameters: $\log (\dot{M})=-9.3, v_{\infty}=1000 \mathrm{kms}^{-1}$. This figure shows that C IV is efficiently destroyed by $\mathrm{X}$-rays in the outer parts of the atmosphere. Without accounting for the ionization by X-rays, the mass-loss rate would be underestimated. Adopted from Oskinova et al. (2011b).

ionization stage. They suggested that the real mass-loss rates are significantly higher, in agreement with the cool optical depth estimated by Waldron and Cassinelli (2007) from the analysis of X-ray lines in O-star spectra.

This idea was tested by NLTE stellar wind models that account for the influence of Xrays on ionization. PoWR models for a sample of supergiants showed that unrealistically high soft X-ray fluxes are required to significantly alter the phosphorus ionization balance (W.-R. Hamann, private communication). Krtička and Kubát (2012) studied the influence of XUV radiation on $\mathrm{PV}$ ionization. They confirmed that strong XUV ra- diation can decreases the $\mathrm{P} \mathrm{V}$ ionization fraction. However, the XUV radiation would also influence the ionization of elements that drive the wind, leading to a decrease of the wind terminal velocity which is not confirmed by observations. Hence, it appears that albeit $\mathrm{X}$-rays influence the ionization structure in the inner wind, they cannot be fully responsible for the low mass-loss rates derived from the analysis of the $\mathrm{P} v$ line.

Oskinova et al. (2007) and Šurlan et al. (2012, and references therein) showed that the "PV problem" is resolved and consistent mass-loss rates are obtained from $\rho^{2}$ and $\rho$-dependent diagnostics when clumping is fully included in the modeling of UV resonance lines. Owocki (2008) and Sundqvist et al. (2010) used an alternative formalism to describe specifically the effects of a non-monotonic velocity and coined the term "vorosity". Using their 2D models, they found that for intermediate strong lines the velocity spans of the clumps are of central importance, albeit density structure and interclump medium also play a role.

Šurlan et al. (2013) performed advance 3D Monte-Carlo simulations of the formation of resonance doublets in a clumped wind. In a detailed parameter study it was demonstrated that "vorosity" has only a moderate effect. In any case, it seems that separating porosity and vorosity effects is rather artificial as both are playing role in establishing the line opacity. The concept of macroclumping (see Section 8) accounts for both of these effects, and consistently includes them in the modeling (e.g. Šurlan et al., 2013). 


\section{The "weak wind problem"}

The mass-loss rates of low luminosity $\left(\log L_{\mathrm{bol}} / L_{\odot}<5.2\right)$ main sequence OB-type stars, e.g. stars with spectral types O9V and later, are orders of magnitude lower than predicted by "standard" mass-loss recipes (Martins et al., 2005; Marcolino et al., 2009; Najarro et al., 2011; Oskinova et al., 2011b). This is often referred in the literature as the weak wind problem.

There are several hypotheses explaining why the mass-loss rates of OB dwarfs empirically derived from UV diagnostics are very low. These hypotheses can be divided into two broad groups: (i) some process reduces the wind driving force in $\mathrm{OB}$ dwarfs making the winds genuinely weak, and (ii) the large fraction of the wind is not visible in the UV lines and therefore cannot be measured using UV diagnostics. These theories are briefly considered below.

\subsection{Do X-rays reduce the wind driving in $O B$ dwarfs and quench the wind?}

According to the standard theory (CAK Castor et al., 1975), stellar winds are radiatively driven. Drew et al. (1994) realized that the presence of $\mathrm{X}$-rays changes the ionization structure in the inner part of the wind. The overionization by X-rays may reduce the total radiative acceleration and lead to a lower mass loss. This suggestion was investigated by Oskinova et al. (2011b) using X-ray observations of a sample of $\mathrm{B}$ dwarfs. The $\mathrm{X}$-rays at the observed level and temperature were included in PoWR stellar atmosphere models, and the wind radiative force was computed.
However, the models did not reveal a significant inhibition of the wind driving power.

On the other hand, Springmann and Pauldrach (1992) questioned the application of the standard radiatively driven wind mechanism for B-type stars. They showed that for low density winds the assumption of a one-component fluid is not valid. In such winds the metal ions loose their dynamical coupling to the ions of hydrogen and helium. The metal ions move with high velocities, while helium and hydrogen are not dragged along. The collisionally induced momentum transfer is accompanied by frictional heating, which dominates the energy balance. As a result, Springmann and Pauldrach (1992) predict electron temperatures of $T \lesssim 1 \mathrm{MK}$ in the outer wind regions for stars with mass-loss rates of $\approx 10^{-8 \ldots 9} M_{\odot} \mathrm{yr}^{-1}$. The subsequent analysis by Krtička and Kubát (2000) and by Owocki and Puls (2002) favored even weaker winds with $\dot{M} \sim 10^{-11} M_{\odot} \mathrm{yr}^{-1}$ for the frictional heating to occur. In this concept, the $\mathrm{X}$-ray emission is not the cause for the small mass-loss rates empirically derived from UV and optical spectra, but its consequence.

Krtička (2014) further elaborated the theory of mass loss from B stars. His theoretical mass-loss rates for main-sequence B-type stars are in good agreement with those empirically derived by Oskinova et al. (2011b). Furthermore, it was shown that at solar abundance, a homogeneous line-driven wind is not possible for stars with spectral types later than B5. This is in agreement with the wind limit found by Abbott (1979). More recently, Muijres et al. (2012) demonstrated that the well established Monte Carlo model 
fails to initiate radiatively driven winds for main-sequence stars with luminosities below $\log \left(L_{\mathrm{bol}} / L_{\odot}\right) \approx 5.2$. Consequently, the standard mass-loss rate recipe (Vink et al., 2001), often used in the literature, may severely overestimate the mass-loss rates of main sequence B stars.

Another possible solution of the weak wind problem was suggested by Martins et al. (2005). If present, a sufficiently strong organized magnetic field could confine the stellar wind (Babel and Montmerle, 1997). The wind streams collide in the equatorial plane where strong shocks produce relatively hard $\mathrm{X}$-rays. The resulting X-rays enhance the ionization in the wind, destroy the lower ions with many lines responsible for wind driving, and thus quench the wind.

Indeed, strong magnetic fields have been detected in some OB dwarfs such as HD 54879 (O9.7V) and HD 57682 (O9IV) (Castro et al., 2015; Grunhut et al., 2009). The latter star has a weak wind and is a hard and luminous X-ray source $(\langle T\rangle \approx 20 \mathrm{MK}$, $\left.\log \left(L_{\mathrm{X}} / L_{\mathrm{bol}}\right) \approx-6\right)$. On the other hand, there are O-type dwarfs where the presence of a strong magnetic field is ruled out, such as $\zeta$ Oph (O9.2IV) and AE Aur (O9.5V) (Schnerr et al., 2008; Hubrig et al., 2011). These objects have weak winds but a normal level of soft X-ray emission not sufficient to quench the winds.

Thus, it appears that at the moment there is no strong support for the idea of the wind quenching in $\mathrm{OB}$ dwarfs.

\subsection{Is the large fraction of $O B$ dwarf winds} in the hot phase?

The theory predicts rather low mass-loss rates for main-sequence B-stars Babel (1996). If a fraction of a B star wind gets heated, e.g. in strong shocks, the cooling time and length may be so long that the bulk of wind material may remain in the hot phase (e.g. Lucy, 1982; Abbott and Friend, 1989). This fraction cannot be detected at optical and UV wavelengths, but is seen in X-rays ${ }^{1}$.

This idea is supported by X-ray observations. Cassinelli et al. (1994, and references therein) noticed that the X-ray emission measure $\left(E M_{\text {hot }}=\int_{V} n_{\mathrm{e}, \mathrm{hot}}^{2} \mathrm{~d} V\right)$ in nearmain-sequence B stars is comparable or even larger than the cool wind emission measure, $E M_{\mathrm{w}}=\int_{V} n_{\mathrm{e}, \mathrm{cool}}^{2} \mathrm{~d} V$ (see also Cohen et al., 2006). This implies that the bulk of stellar wind matter may be in a hot phase. This hot fraction of the wind material is "missed" when mass-loss rates are empirically deduced from the emission of the cool wind observed as an excess in the infrared or radio continuum, or from P Cygni line profiles in the UV.

Lucy (2012) suggested that in late-type O dwarfs the shock-heating of the ambient gas increases its temperature, and the resulting single-component flow with a temperature of a few MK coasts to high velocities as a pure coronal wind. Hence, the volumetric roles of hot and cool gas are reversed in $\mathrm{O}$ dwarfs compared to $\mathrm{O}$ supergiants. The bulk of

\footnotetext{
${ }^{1}$ Note that radio and IR observations may provide additional constraints, as shown in Lamers and Cassinelli (1999, p.29)
} 
wind is hot, while it contains only some cold clumps.

X-ray observations of O-dwarfs seem to support this scenario. High-resolution X-ray spectra of $\zeta \mathrm{Oph}(\mathrm{O} 9.2 \mathrm{IV}), \mu \mathrm{Col}$ $(09.5 \mathrm{~V})$, AEAur $(09.5 \mathrm{~V})$, and $\sigma$ Ori AB $(\mathrm{O} 9.5 \mathrm{~V}+\mathrm{B} 0.5 \mathrm{~V})$ show similar morphology (Oskinova et al., 2006; Skinner et al., 2008; Huenemoerder et al., 2012). The X-ray luminosity of $\mathrm{O}$ dwarfs is a significant fraction of the cool wind's mechanical luminosity. For instance, the ratio between $L_{\mathrm{X}}$ and $L_{\text {wind }}=0.5 \dot{M} v_{\infty}^{2}$ amounts to 0.1 in $\mu \mathrm{Col}$ and 0.7 in AE Aur. There is only limited evidence for an absorption of X-rays by the cool wind component, consistent with the above picture. Analyses of line ratios of He-like ions (see Section 3) indicate that the hot matter is present already in inner wind regions where the wind velocity is low. The X-ray emission lines are broadened up to $v_{\infty}$, and can be well described as originating from a plasma expanding with a $\beta$-velocity law, i.e. the hot plasma occupies a large span of velocities.

Thus, it seems that a new picture of an OB dwarf stellar wind is emerging from theory and observation. The winds of O-dwarfs are predominantly in the hot phase, while the cool gas seen in the optical and UV constitutes only a minor wind fraction. Hence, the best observational window for studies of OBdwarfs is provided by X-rays.

\section{B-dwarfs in the X-ray light}

As outlined above, X-rays provide an important window to study winds of nonsupergiant B-type stars that are hardly ac- cessible in other bands of the electromagnetic spectrum. However, high quality X-ray data exist for only a handful of non-supergiant Btype stars, and these are observationally biased to the closest and most intriguing objects, e.g. magnetic stars or binaries.

A report on the diverse X-ray properties of some magnetic B dwarfs can be found in Oskinova et al. (e.g. 2011b); Nazé et al. (e.g. 2014). B-type binaries also have diverse $\mathrm{X}$ ray characteristics. $\theta$ Car $(\mathrm{B} 0.2 \mathrm{~V})$ is an $\mathrm{X}$ ray luminous $\left(\sim 10^{31} \mathrm{erg} \mathrm{s}^{-1}\right)$ binary with soft X-ray spectrum (Hubrig et al., 2008; Nazé and Rauw, 2008). On the other hand, $\rho$ Oph $(\mathrm{B} 2 \mathrm{IV}+\mathrm{B} 2 \mathrm{~V})$ has a quite hard variable $\mathrm{X}$-ray spectrum (Pillitteri et al., 2014).

Perhaps the B-type star studied best in $\mathrm{X}$-ray is the early-type dwarf $\tau$ Sco $(\mathrm{B} 0.2 \mathrm{~V})$ (Howk et al., 2000). Mewe et al. (2003) obtained its first high-resolution X-ray spectrum. Its analysis yielded temperatures in the range of $1 . .20 \mathrm{MK}$. The emission measure of the hot plasma is comparable to that of the cool wind. It was found that the spectral lines are not broadened above the instrumental profile. Correspondingly, the X-ray emitting plasma does not expand faster than with $\sim 500 \mathrm{~km} \mathrm{~s}^{-1}$, i.e. it is much slower than the cool wind that has a velocity of $v_{\infty} \sim 1000 \mathrm{~km} \mathrm{~s}^{-1}$ (Oskinova et al., 2011b). From the analysis of line ratios in the emission of He-like ions it was shown that the Xray emission originates from relatively close to the stellar surface (see also Cohen et al., 2003).

Donati et al. (2006) discovered a magnetic field on $\tau$ Sco with a complex topology. This field should be capable to confine at least 
some portion of the stellar wind and, in principle, may help to explain the characteristic shapes of the UV lines. It was suggested that the X-ray emission predominantly originates from closed magnetic loops that are distributed non-uniformly over the stellar surface. Because of rotation, the active regions cross our line of sight. If produced in these active regions, the rotational modulations of X-rays should be up to $40 \%$ (Donati et al., 2006). Surprisingly, monitoring of $\tau$ Sco over its rotation period with the Suzaku X-ray observatory did not reveal the predicted variability, indicating that the X-rays are likely produced in small-scale magnetic loops distributed across the whole stellar surface (Ignace et al., 2010).

Recently, it was confirmed that a whole group of stars that have UV spectral features similar to $\tau$ Sco also host magnetic fields; these stars, HD 66665 and HD 63425, were dubbed $\tau$ Sco-analogs (Petit et al., 2011). Ignace et al. (2013b) investigated their X-ray properties, and found them similar to those of $\tau$ Sco. Thus, it appears that $\tau$ Sco analogs have similar magnetic, stellar wind, and coronal properties.

The sample of non-magnetic B-dwarfs studied in X-rays is rather small. The star $\sigma \operatorname{Sgr}(\mathrm{B} 2.5 \mathrm{~V})$ was only marginally detected by $X M M-N e w t o n$ in a $10 \mathrm{ks}$ exposure. Its X-ray luminosity $L_{\mathrm{X}} \approx 4 \times 10^{27} \mathrm{erg} \mathrm{s}^{-1}$ $\left(\log L_{\mathrm{X}} / L_{\mathrm{bol}} \approx-9.4\right)$ (Oskinova et al.in prep) is much lower than previously reported from Rosat observations. Possibly, the latter measurement was contaminated by a close and X-ray variable low-mass coronal companion (Gullikson and Dodson-Robinson, 2013).
Active low-mass stars may often outshine their more massive companions in the X-ray light (e.g. Evans et al., 2011).

\subsection{Pulsating $\beta$ Cep-type stars}

Young hydrogen-burning B0-B2 type stars pulsate with periods of a few hours. These stars are called $\beta$ Cep-type variables. The oscillations are driven by changes of the opacity inside the star during the pulsation cycle (" $\kappa$-mechanism", Dziembowski and Pamiatnykh, 1993). Like other early B-type stars, $\beta$ Cep-type variables are X-ray sources.

Besides radiatively driven stellar wind and magnetic effects, stellar pulsations may play a role in powering the X-ray emission from B stars. E.g., Neilson and Lester (2008) and Engle et al. (2014) demonstrated that pulsations may heat the outer atmosphere of classical Cepheids and power X-ray emission even in these cool stars.

Observations with the EUVE satellite showed that $\beta \mathrm{CMa}$ (B1II/III) and $\epsilon \mathrm{CMa}$ (B1.5II) have an order of magnitude excess in their extreme UV (EUV) spectra relative to the predictions of stellar atmosphere models. It was suggested that the deposition of mechanical energy from stellar pulsations can heat the inner wind regions causing the observed excess (Cassinelli et al., 1996). W.R. Hamann (priv. communication) showed that the observed extreme UV excess in $\beta$ and $\epsilon$ CMa could be reproduced by accounting for a continuous temperature distribution of the X-ray emitting plasma through shock heating and the subsequent cooling sequence. Interestingly, Fossati et al. (2015) detected 
weak magnetic fields on $\beta \mathrm{CMa}$ (B1II/III) and $\epsilon$ CMa (B1.5II).

If stellar oscillations are somehow responsible for the X-ray emission from $\beta$ Cep variables, one may hope to find correlations between the pulsational behavior and the $\mathrm{X}$ ray properties. An initial X-ray survey of six $\beta$ Cep-type stars was performed with the Einstein observatory (Agrawal et al., 1984), but no correlations were found between X-ray and pulsational, rotational, or binary properties. Negative results were also obtained in the study of four $\beta$ Cep stars observed with Rosat (Cassinelli et al., 1994). Oskinova et al. (2011b) searched for correlations between pulsational and X-ray properties in a small sample of $\beta$ Cep-variables with well known pulsational behavior and existing Xray observations, but did not find any. Timing analyses of $\beta$ Cen and $\beta$ Cep using very high quality X-ray light curves obtained with $X M M-N e w t o n$ and Chandra did not reveal any X-ray oscillations (Raassen et al., 2005; Favata et al., 2009). Both these stars are magnetic (Donati et al., 2001; Schnerr et al., 2006; Alecian et al., 2011). A report on detected X-ray pulsations of $\beta$ Cru (Cohen et al., 2006) could not be confirmed by an independent study (Oskinova et al., 2015).

Stable, coherent X-ray pulsations in phase with the optical light-curve but even larger amplitude were firmly identified so far only in one B type star $-\xi^{1}$ CMa (B0.7IV) (Oskinova et al., 2014). This star has the strongest magnetic field among all $\beta$ Cep-type variables with available measurements (Hubrig et al., 2006). The phase resolved X-ray spectroscopy using XMM-Newton data revealed that the X-rays originate from close to the stellar photosphere, questioning the prediction of the magnetically confine wind shock model (Babel and Montmerle, 1997, for the latest development of the model see the review of ud-Doula \& Nazé in this volume).

\section{X-ray variability as a diagnostic of inhomogeneous stellar winds}

Obtaining X-ray light curves and establishing parameters of X-ray variability provides a valuable tool to gain insight into the stellar wind structure.

There are numerous observational evidences supported by theoretical considerations for stellar winds being structured and inhomogeneous (e.g. Hamann et al., 2008). Among the first clear demonstrations of wind inhomogeneity was the detection of stochastic variability in the He II $\lambda 4686 \AA$ emission line in the spectrum of an $\mathrm{O}$ supergiant (Eversberg et al., 1998), explained by clump propagation. Markova et al. (2005) investigated the line-profile variability of $\mathrm{H} \alpha$ for a large sample of O-type supergiants and concluded that the observed variability can be explained by a structured wind consisting of shells fragments. Using spectral diagnostics Prinja and Massa (2010) showed that the winds of B supergiants are clumped. In a recent study Martins et al. (2015) found that spectral lines formed in the winds of all OB supergiants in their sample are variable on various time scales. Lépine and Moffat $(1999,2008)$ monitored the line-profile variations in a sample of WR and O stars and explained their observations using a phenomenological model 
that depicts winds as being made up of a large number of randomly distributed, radially propagating clumps.

Convincing evidence of wind clumping is provided by high-mass X-ray binaries (HMXBs). In some of these systems, a neutron star (NS) is in a close orbit deeply inside the stellar wind of an OB star. Accretion from the clumped stellar wind onto the NS powers strongly variable X-ray emission (e.g. Bozzo et al., 2011; Oskinova et al., 2012; Martínez-Núñez et al., 2014). van der Meer et al. (2005) studied the X-ray light curve and spectra of $4 \mathrm{U}$ 1700-37 and concluded that the feeding of the NS by a strongly clumped stellar wind is consistent with the observed stochastic variability. Further evidence of donor wind clumping comes from the analysis of the X-ray spectra. Schulz et al. (2002); Giménez-García et al. (2015) reviewed the spectroscopic results obtained with X-ray observatories for wind-fed HMXBs. They explained the observed spectra as originating in a clumped stellar wind, where cool dense clumps are embedded in rarefied photoionized gas. Torrejón et al. (2015) used fluorescence lines as a tracer of wind clumps and determined that these clumps must be present close to the photosphere in case of a B-type supergiant.

One can expect that, similar to the variability observed in optical and UV light, the $\mathrm{X}$-ray emission from OB supergiants should also be variable. Indeed, hydrodynamic models predict strong stochastic X-ray variability with very large amplitude, albeit this may be an artifact of the 1-D geometry of hydrodynamic simulations (Feldmeier et al., 1997a,b).
Yet, strong stochastic X-ray variability is not observed. Oskinova et al. (2001) developed a phenomenological wind model and showed that the character of X-ray variability depends on the number of shocks as well as on the cool wind opacity. Consequently, the observations of X-ray variability can provide important information on the structure of stellar wind.

Given its large diagnostic potential, there were numerous attempts to measure the $\mathrm{X}$ ray variability of $\mathrm{O}$ stars. Berghoefer et al. (1996) used the X-ray telescope Rosat (0.9$2.4 \mathrm{keV}$ ) to monitor $\zeta$ Pup over 11 days totaling 56 ks observing time. Contemporary with $\mathrm{X}$-rays the variability of $\mathrm{H} \alpha$ was also monitored. They reported a $16.667 \mathrm{~h}$ modulation in the $\mathrm{H} \alpha$ line correlated with modulations in the X-rays. The amplitude of the X-ray variability was found to be $\pm 6 \%$. These results were interpreted as an evidence for periodic variations in the wind density.

Oskinova et al. (2001) analyzed archival X-ray observations of $\zeta$ Pup and $\zeta$ Oph made with the X-ray telescope ASCA (0.5-10 $\mathrm{keV})$. ASCA observed $\zeta$ Pup continuously for $28.4 \mathrm{~h}$. The timing analysis of the data did not reveal modulations as those reported by Berghoefer et al. (1996). On the other hand, modulations on the time scale of days were found in the X-ray emission of $\zeta$ Oph.

Massa et al. (2014) analyzed X-ray observations of $\xi$ Per (O7.5III) obtained with the Chandra X-ray telescope and contemporaneous $\mathrm{H} \alpha$ observations. The X-ray flux was found to vary by $\sim 15 \%$, but not in phase with the $\mathrm{H} \alpha$ variability. The observations were not long enough to establish periodic- 
ity.

Among the longest time intervals covered by X-ray observations of a particular star is the monitoring of $\zeta$ Pup by XMM-Newton. About 20 exposures of various duration were obtained during a decade. Timing analysis of these observations did not reveal the period reported by Berghoefer et al. (1996). However, variations with amplitude of $\sim 15 \%$ on a time scale longer than $1 \mathrm{~d}$ were found, while no coherent periodicity was detected (Nazé et al., 2013).

Rauw et al. (2015a) obtained X-ray and coordinated $\mathrm{H} \alpha$ observations of $\lambda$ Cep $(\mathrm{O} 6.5 \mathrm{I}(\mathrm{n}))$. They found that the X-ray flux varies by $\sim 10 \%$ on timescales of days and might be modulated by the same period as the $\mathrm{H} \alpha$ emission, but shifted in phase. The analysis of archival XMM-Newton observations of $\zeta$ Ori shows that the X-ray variability of this star has similar properties to that of $\lambda$ Cep and $\zeta$ Pup. X-ray variability with analogous character was found in $\delta$ Ori $(\mathrm{O} 9.5 \mathrm{II}+\mathrm{B} 1 \mathrm{~V})$ from Chandra observations, where periodic X-ray fluctuations (not associated with the binary period) were identified (Nichols et al., 2015). Even WR stars with very strong winds show modulations in their X-ray emission on the rotation timescale (Ignace et al., 2013a).

Thus, solid evidence of slow X-ray variability of O-type star is accumulating. The new high-quality data suggest an association between X-ray emission and large scale structures in the stellar wind.

It seems, that the X-ray variability is closely related to the coherent and periodic variability that is commonly observed in the
UV spectral lines of OB supergiants (e.g. Kaper et al., 1999; Massa and Prinja, 2015). The latter is explained by the existence of corotating interaction regions (CIRs) in stellar winds, which can also play a role in the generation of X-rays (Mullan, 1984). Cranmer and Owocki (1996) showed that CIRs could result from bright stellar spots. Ramiaramanantsoa et al. (2014) detected small corotating bright spots on $\xi$ Per and suggested that they are generated via a breakout of a global magnetic field generated by subsurface convection. The CIRs may also be triggered by the (non)radial pulsations of the stellar surface (Lobel and Blomme, 2008).

\section{Modeling of X-ray emission line pro- files}

In this section, the diagnostic power of $\mathrm{X}$ ray emission lines to probe stellar winds is considered. Presently, lines are resolved in the X-ray spectra of nearly all types of massive stars. Line shapes and fluxes provide valuable information about the motion and geometrical distribution of the hot plasma, and allow to probe stellar wind opacity. In general, the X-ray emission lines are broad, indicating that the hot plasma is expanding with high velocities, comparable to the wind velocity obtained from UV line measurements (e.g Waldron and Cassinelli, 2007). Moreover, the shape of the emission lines in $\mathrm{O}$ star spectra shows that the hot plasma is spread over a large range in the velocity space (e.g. Corcoran et al., 2015)

The shape of an X-ray emission line can be calculated from a solution of radiative trans- 
fer equation describing the emission from expanding optically thin shell and its absorption in the smooth cool stellar wind with high continuum opacity (Macfarlane et al., 1991).

The optical depth along the radial line of sight is given by

$$
\tau_{\lambda}=\int_{r_{0}}^{\infty} \chi_{\lambda}(r) \mathrm{d} r
$$

where $r_{0}$ is expressed in the units $R_{*}$ and denotes the onset of X-ray emission, and the atomic opacity

$$
\chi_{\lambda}=\rho_{\mathrm{w}} \kappa_{\lambda}
$$

is the product of the mass absorption coefficient $\kappa_{\lambda}\left[\mathrm{cm}^{2} \mathrm{~g}^{-1}\right]$ and the density of the cool wind $\left(\rho_{\mathrm{w}}\right)$. The latter obeys the continuity equation

$$
\dot{M}=4 \pi \rho_{\mathrm{w}}(r) v(r) r^{2} R_{*}^{2},
$$

where $r$ is the radial coordinate in the units of $R_{*}$, and $v(r)$ is the velocity law. Commonly, a $\beta$-velocity law is adopted

$$
v(r)=v_{\infty}\left(1-\frac{1}{r}\right)^{\beta} .
$$

Macfarlane et al. (1991) applied their formalism to the very soft X-ray (XUV) radiation of $\mathrm{B}$ supergiants. They reasoned that because the stellar wind is quite opaque at these wavelengths, the observed radiation must originate from outer wind regions, where the wind ionization is constant. In this case one can neglect the radial dependence of the mass absorption coefficient $\kappa_{\lambda}$ and Eq. (1) can be simplified to

$$
\tau_{\lambda}=\tau_{*} \int_{R_{0}}^{\infty}\left(1-\frac{r_{0}}{r}\right)^{-\beta} r^{-2} \mathrm{~d} r,
$$

where

$$
\tau_{*}=\frac{\kappa_{\lambda} \dot{M}}{4 \pi v_{\infty} R_{*}} .
$$

Macfarlane et al. (1991) pointed out that the line shape is largely determined by the parameter $\tau_{*}$. When $\tau_{*}$ is small, the line is broad and has a box-like shape. For stronger wind absorption, the line becomes more skewed (see Figure 7 in Macfarlane et al., 1991), because the photons in the red-shifted part of the line are more attenuated than in the blueshifted part. Macfarlane et al. (1991) showed that evaluating the line shape can be used to determine $\tau_{*}$, and thus probe the wind opacity.

The opacity for X-rays is largely determined by the K-shell absorption in metal ions. The K-shell opacities, and consequently the coefficient $\kappa_{\lambda}$, vary with wavelength with a power between 2 and 3 (Verner and Yakovlev, 1995). Therefore, $\tau_{*}$ should change by orders of magnitude over the Xray band $(\sim 1-30 \AA)$. Thus, the shape of emission lines should be different at longer and at shorter wavelengths.

The Macfarlane et al. (1991) formalism turned out to be extremely useful in describing the X-ray emission line profiles when the first high-resolution X-ray spectra of OB stars were obtained with XMM-Newton and Chandra. Contrary to the predictions, the observed X-ray lines showed only little asymmetry, and were found to have similar shapes at different wavelengths (Waldron and Cassinelli, 2001; Kahn et al., 2001; Cassinelli et al., 2001).

Waldron and Cassinelli (2001) consid- 
ered emission from spherically symmetric shocks equally distributed between $0.4 v_{\infty}$ and $0.97 v_{\infty}$ with temperatures ranging from 2 to $10 \mathrm{MK}$. They found that the mass-loss rate of the O-type supergiant $\zeta$ Ori must be lower than traditionally assumed in order to explain the observed nearly symmetric X-ray emission line profiles.

Owocki and Cohen (2001) updated the formalism from Macfarlane et al. (1991) by including a $\beta$-velocity law and allowing for a radial dependence of the hot gas filling factor. Independently, Ignace (2001) considered expansion with a constant velocity but included a radial dependence of the filling factor.

\subsection{X-ray line profiles from clumped stellar winds}

Stellar wind clumping was included in the line formation formalism by Feldmeier et al. (2003). The stellar wind structure affects the propagation of $\mathrm{X}$-rays and the emergent X-ray line profile. In a first approximation ("microclumping"), clumping is taken into account in up-to-date stellar wind codes (Hillier, 1991; Hamann and Koesterke, 1998; Puls et al., 2006). This approximation assumes that all clumps are optically thin. The interclump medium is void or filled with tenuous matter (Zsargó et al., 2008). In this socalled microclumping approximation the radiative transfer is not affected by the clumping, which makes this approximation very convenient. One usually introduces a parameter $D$ to describe the factor by which the density in the clumps is enhanced compared to a homogeneous wind with the same massloss rate. If the interclumped medium is as-

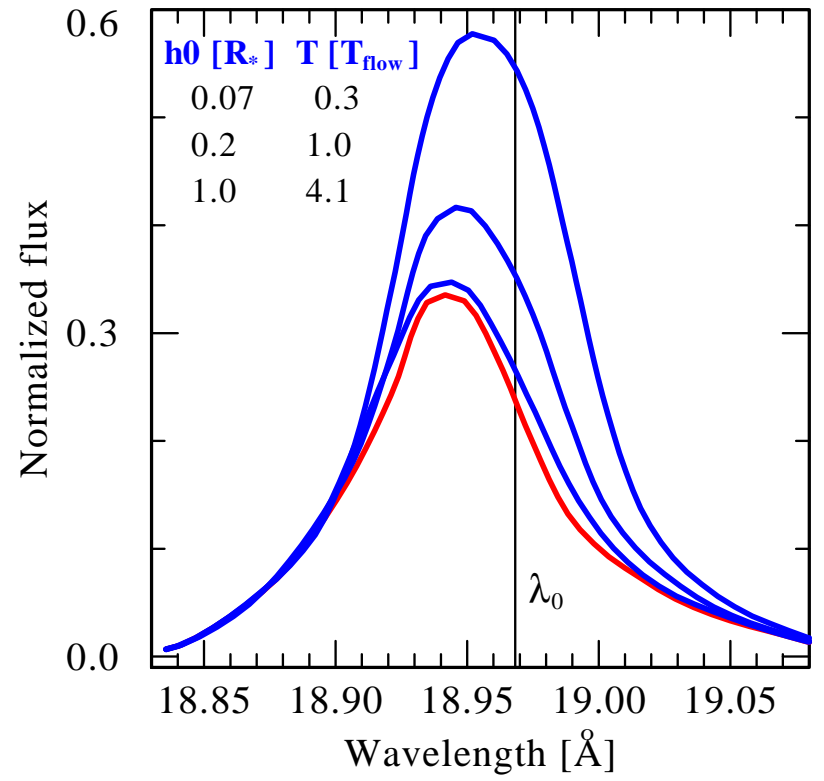

Figure 4: The effect of clumping parameter $T$ (or $h\left(R_{0}\right)$ ) on model lines assuming anisotropic opacity with clumps flattened in radial direction, e.g. broken shell fragments. Except of the clumping parameter $T$ $\left(h\left(R_{0}\right)\right)$, model parameters are for the star $\delta$ Ori and are the same for each model line. The red line is for smooth wind, while blue lines are for clumped wind models with porosity length $h\left(R_{0}\right)<1$. The line computed with the smallest porosity length is similar to the smooth wind model.

sumed to be void, the clump volume filling factor is $f_{\mathrm{V}}=D^{-1}$. The most important consequence of microclumping is the reduction of mass-loss rates empirically derived from diagnostics that depend on density squared (such as $\mathrm{H} \alpha$ emission fed by the recombination cascade, and free-free radio emission) by a factor $\sqrt{D}$ (e.g. Hamann and Koesterke, 1998; Bouret et al., 2005; Fullerton et al., 2006).

Microclumping is a stringent approximation, and one has to be aware of its limitations. Waiving the microclumping ap- 
proximation in modeling is called "macroclumping". Macroclumping does not impose any restrictions on a clump's optical depth. The same clump could be optically thin at some wavelength and optically thick at another. This has implications for the analysis of X-ray spectra from OB supergiants - the lines at shorter wavelengths may not be affected, while clumping effects become more pronounced for lines at longer wavelengths (Oskinova et al., 2006). Ignace (2016) studied the influence of macroclumping on the free-free spectral energy distribution of ionized winds, with applications to the formation of radio spectra and to mass-loss rate diagnostics.

A general theory for the transfer of Xray radiation in clumped stellar winds, including limiting cases of optically thin and fully opaque clumps, as well as intermediate cases, was developed in a series of papers by Feldmeier et al. (2003); Oskinova et al. (2004, 2006). An analytic description for the effective opacity in isotropic and anisotropic cases was found. (Figure 4 demonstrates lines emerging from winds with anisotropic opacity.) It was pointed out that in case of isotropic opacity (e.g. spherical clumps) the line profiles are identical to those emerging from a smooth wind. On the other hand, in case of anisotropic opacity, the line profiles are characteristically different. The analytic solutions were verified by 2.5-D Monte-Carlo simulations (Oskinova et al., 2004), and reproduced by, e.g., Sundqvist et al. (2012) using their alternative approach. Detailed considerations of X-ray line formation in stellar winds are given in the review by $\mathrm{R}$. Ignace (this volume).

To specify clumping properties, Oskinova et al. (2001, 2004) used a "fragmentation frequency" parameter $n$. One can envision this as the number of clumps that are "launched" from some initial radius per dynamical time $T_{\mathrm{fl}}=R_{*} / v_{\infty}$. Assuming that clumps do not merge and do not disappear until some outer boundary, the fragmentation frequency $n$ is radius independent. The inverse of the fragmentation frequency, $T=1 / n$, is the average time between two successive clumps passing through some point in the wind.

Owocki and Cohen (2006) and Leutenegger et al. (2013) used the "porosity" formalism to model X-ray emission lines emerging from clumped winds. Hervé et al. (2012) provided a careful comparison of the macroclumping and porosity formalisms and concluded that they are "essentially equivalent to first order".

Owocki et al. (2004) and Owocki and Cohen (2006) use the porosity length $h$ to parametrize the clumping properties. This porosity length is the ratio of the volume per blob to the projected surface area of the blob, $h=L^{3} / l^{2}$. Equivalently, it is the blob size divided by its volume filling factor, $h=l /(l / L)^{3}$ (Owocki et al., 2004). Usually, it is assumed that the porosity growth with the radial coordinate and reaches its maximum value at $v_{\infty}$. Owocki and Cohen (2006) argued that porosity effects become important only for unrealistically large porosity length $h \gg 1$. However, Oskinova et al. (2006) showed that because of the strong wavelength dependence of the wind opacity, porosity effects are also strongly wavelengths 
dependent. As illustrated in Fig 6, realistically small porosity lengths are sufficient to influence X-ray lines.

In the hydrodynamic models by Feldmeier et al. (1997b) the X-rays are generated when a fast parcel of gas rams into a slower moving dense shell. Feldmeier et al. (2003) solved the X-ray radiative transfer in such situation, and showed that the emerging line profiles have a strong characteristic dip at the line center. In the hydrodynamic models by Ignace et al. (2010) the X-rays are emitted from bow shocks created by dense clumps. These models also predict a dip in the center of the emergent line

The quality of available X-ray spectra allows to study fine details of X-ray line profiles. It appears that a central dip is indeed observed in some strong X-ray lines (see e.g. Fig. 5). Alternative explanations of the central dip may include resonance scattering (Oskinova, 2006). Profile shapes for optically thick X-ray emission lines from stellar winds were modeled by Ignace and Gayley (2002).

\section{Using X-ray spectra to probe stellar wind opacity and mass-loss}

The winds of OB supergiants are strong and efficient in absorbing X-rays. Therefore, the X-ray spectra could be exploited to provide useful information on wind column density, abundances, and structure.

Waldron (1984) used calculated wind opacities and demonstrated that the analysis of X-ray spectra can be used to probe the latter. To describe the propagation of broad-band X-ray radiation in stellar winds,

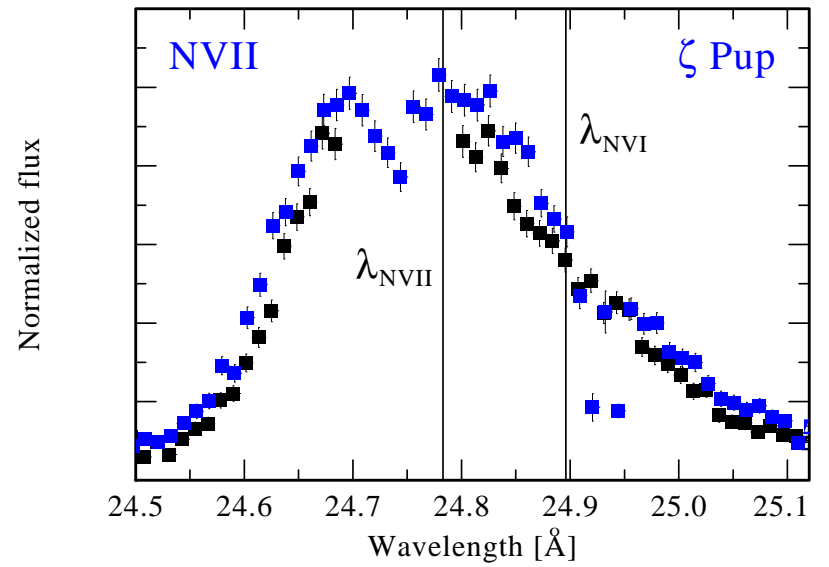

Figure 5: The N VII $\lambda 24.758 \AA$ and N VI $\lambda 24.898 \AA$ blend in the XMM-Newton RGS1 (blue squares) and RGS2 (black squares) spectra of $\zeta$ Pup. The central wavelengths of the lines are indicated. The absorption dip at $\approx 24.75 \AA$ is characteristic for nonnegligible line optical depth. Figure produced from archival data.

Owocki and Cohen (1999), Ignace and Oskinova (1999), Ignace et al. (2000) adopted the exospheric approximation in which all of the emission from above the sphere with the radius where the optical depth is unity escapes, while all emission from below this radius is absorbed. This is a reasonable approximation because the "contribution function" to a line peaks at the radius where wind optical depth is close to unity. A more precise solution was presented by Leutenegger et al. (2010).

Hillier et al. (1993) computed the cool wind opacity of $\zeta$ Pup assuming a smooth wind and applied their model to the low resolution Rosat X-ray spectrum. It was found that the high opacity of the stellar wind should completely block the soft X-rays $(<0.5 \mathrm{keV})$. However, since such soft X-rays 
are observed, it was concluded that a significant fraction of the hot plasma is located far out in the wind, at distances exceeding $100 R_{*}$.

However, the analysis of high-resolution $\mathrm{X}$-ray spectra of $\mathrm{O}$ stars (see Section 3) revealed that the $\mathrm{X}$-rays are produced close to the photosphere. The question how those Xrays could pass through the overlaying stellar wind without being completely absorbed has been subject of intensive studies over the past fifteen years.

At least two explanations for the effectively low wind opacity for X-rays were suggested: porosity of a clumped stellar wind (Feldmeier et al., 2003) and lower mass-loss rates than commonly adopted (Kramer et al., 2003).

Besides these explanations, a nonsymmetric two-component wind structure was considered by Mullan and Waldron (2006). They suggested a scenario where one wind component emerges from magnetically active polar regions, while the second, radiatively driven component, originates in a broad range of latitudes centered on the equator. Yet another idea was suggested by Pollock (2007) who proposed that X-rays originate in the wind's terminal velocity regime in collisionless shocks controlled by magnetic fields rather than in cooling shocks in the acceleration zone.

Zhekov and Palla (2007) analyzed the spectra of 15 massive $\mathrm{OB}$ stars and found that their sample stars fall into two groups: stars with relatively soft and with relatively hard X-ray spectra. Zhekov and Palla (2007) cautioned that the origin of $\mathrm{X}$-ray emission is likely different between these groups. While $\mathrm{X}$-rays may originate from small-scale wind shocks in stars with softer spectra, magnetically confined wind shocks are likely to be responsible for the X-ray emission of the other group.

\subsection{Measuring mass-loss rates using X-ray line profiles?}

Recently, it was suggested that a star's mass-loss rate can be determined by fitting the simple quantitative model of Owocki and Cohen (2001) to each emission line in its (Xray) spectrum and then analysing the ensemble of line optical depth (Cohen et al., 2011). If valid, this method would provide an interesting diagnostic of stellar mass-loss rates. However, the method is based on the assumption that the absolute values for the wind opacity derived by Macfarlane et al. (1994) are universal for all O-type stars (Cohen et al., 2014).

The universality of wind opacity is a stringent assumption. It assumes that all O-type stars have the same - solar - chemical composition, and the same ionization stratification of their winds. Yet, detail non-LTE calculations of wind opacities show strong departures from these universal values. For instance, Hervé et al. (2012) computed detailed stellar atmosphere models of $\zeta$ Pup. At $\lambda=19 \AA$ their models predict a factor of $\sim 2$ larger $\kappa_{\lambda}$ than the universal value. These uncertainty would directly propagate to the estimated mass-loss rate. Similarly, Carneiro et al. (2016) recently discussed in detail the behavior of the mass-absorption coefficient in O star winds. Their detailed models of wind 
opacites do not comply with a generic universal values of $\kappa_{\lambda}$ for all $\mathrm{O}$ stars.

Wind clumping and radial dependence of filling factor, which are neglected in Cohen et al. (2014)'s method, also may skew the mass-loss diagnostics. By fitting observed X-ray line profiles, one principally cannot distinguish between clumped models with isotropic opacity and smooth wind models (see Section 8.1). Therefore, the good fits of observed line profiles obtained with smooth wind models indicate at best, that the stellar wind clumps are more or less isotropic in shape (i.e. spherical or randomly oriented).

To test how much clumping affects the mass-loss estimates, we computed a grid of model lines with various clumping parameters, ranging from very strong clumping to smooth winds. As an example, Figure 6 shows two similar model lines, one from emerging from a smooth, and another one from a clumped wind. The figure illustrates that fitting of the line profile, in principle, cannot provide clumping independent massloss rate diagnostics. Even a small porosity length $h$ or high fragmentation frequency $n$ (see Section 8.1) gives a factor of two different mass-loss rate when the latter is evaluated from fitting the line shapes.

Finally, the detailed stellar wind modeling shows that the observed X-ray spectra cannot be reproduced consistently with UV and optical spectra unless either macroclumping or a radial dependence of the hot gas filling factor is included in the model atmospheres (Hervé et al., 2013; Shenar et al., 2015; Puebla et al., 2016).

Thus, measuring stellar mass-loss rate by

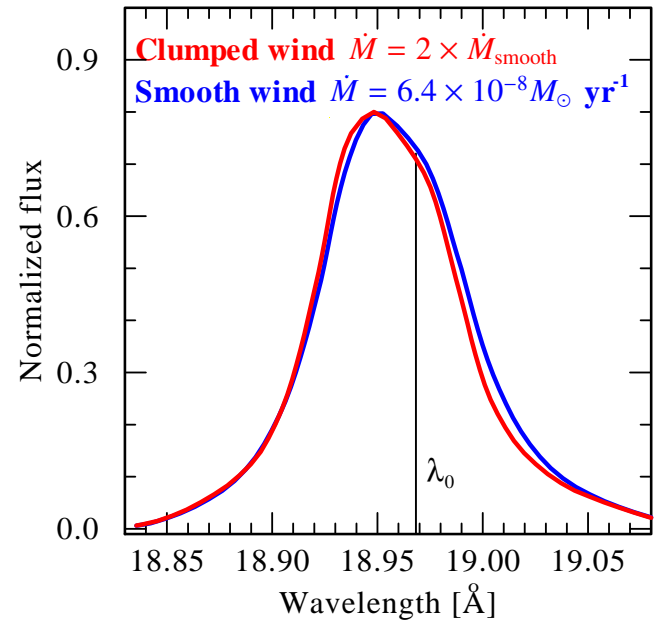

Figure 6: X-ray model line profiles. The vertical line indicates the central wavelength of the $\mathrm{O}$ VIII $\mathrm{L} \alpha$ line. Two model lines computed with identical parameters except of mass-loss rate and clumping are shown. The universal mass-absorption coefficient $\kappa$ is adopted from Cohen et al. (2014). The blue line is for a smooth wind with an onset of X-ray emission at $R_{0}=1.33 R_{*}$ and $\dot{M}=6.3 \times 10^{-8} M_{\odot} \mathrm{yr}^{-1}$. The red line has all the same parameters except that macroclumping with isotropic opacity (spherical clumps) is included and the mass-loss rate is two times larger than for the smooth wind model. The clumped wind model corresponds to a porosity length of $h\left(R_{0}\right)=0.1$. The model lines are convolved with Chandra's MEG spectral resolution.

fitting simplified models to the observed Xray spectra seems to suffer from a parameter degeneracy (it represents an ill-posed inverse problem). Mass-loss rates cannot be reliably derived from the ensemble of X-ray line profiles without additional information on ionization structure, abundances, clumping, and velocity law in stellar wind. 
10. Combining X-ray spectroscopy with multiwavelength analysis to empirically constrain stellar wind properties.

Over the last decade X-ray spectroscopy was successfully combined with traditional methods of spectroscopic analysis. The conditions in stellar winds strongly depart from LTE, and sophisticated codes have been developed to tackle the problem of spectrum formation in this situation. The challenge is to include an X-ray radiation field in these stellar atmosphere models and to obtain a synthetic X-ray spectrum consistently with UV and optical spectra that can be compared with observations.

The PoWR non-LTE stellar atmosphere model accounts for the effect of the X-ray field on the cool wind. Shenar et al. (2015) used PoWR models to study X-ray, optical, and UV spectra of the O star $\delta$ Ori Aa (O9.5II). They adopted a radius dependent clump volume filling factor, with the maximum value $f_{\infty}=0.1$ reached at $\sim 10 R_{*}$ as motivated by hydrodynamic wind simulations. Using macroclumping it was possible to reproduce the UV and optical spectra as well as the X-ray emission lines consistently. The stellar mass-loss was found to be in good agreement with hydrodynamical predictions (Vink et al., 2001).

Hervé et al. (2012, 2013) and Rauw et al. (2015a) included the X-ray radiation in the non-LTE CMFGEN stellar atmosphere models to obtain synthetic high resolution Xray spectrum. The best agreement between the model and the observations was achieved with a non-monotonic radial distribution of the X-ray filling factors and the location of the X-ray plasma in $\lambda$ Cep (O6.5I) between $1.1 R_{*}$ and $2.5 R_{*}$, and in $\zeta$ Pup between $1.5 R_{*}$ and the outer wind regions. Global fits of the X-ray spectra yielded mass-loss rates in very good agreement with previous determinations based on optical and UV spectra.

A consistent analysis of the X-ray, UV, and optical data of the B0Ia supergiant $\epsilon$ Ori was performed by Puebla et al. (2016) using the CMFGEN code. A radial dependence of the X-ray filling factor was used as free parameter to bring the model spectrum in accordance with observations. (Note, however, that Cohen et al. (2010) tested models with varying filling factors by fitting them to observed X-ray emission lines and concluded that a radial dependence of the filling factor can be neglected since this does not improve the line fits.) Macroclumping was not included in the models, but very strong microclumping with $f_{\infty}<0.01$ was required to reproduce the UV spectrum of Si IV line. Under these assumptions, the mass-loss rate would be $\dot{M}<1 \times 10^{-7} M_{\odot} \mathrm{yr}^{-1}$.

\section{X-ray diagnostics of massive stars in the latest stages of their evolu- tion: RSG, WR, and LBV stars}

In its late evolutionary stage, a massive star could become a red supergiant (RSG), luminous blue variable (LBV), or/and a Wolf-Rayet (WR) star. The exact evolutionary path depends on various parameters, such as initial mass, rotation, metallicity, and 
binarity (e.g. Hamann et al., 2006; Langer, 2012; Sander et al., 2012).

Red supergiants are X-ray dark. Searches with XMM-Newton for X-ray emission possibly associated with surface magnetic activity or shocks in the RSG winds resulted in null detections (Montez et al., 2015, and references therein). The winds of RSGs are strong, therefore the null result may not be surprising, even if any X-ray emission were generated in lower wind layers, it would be absorbed in the wind. 3D hydrodynamic models predict that in binary systems, such as e.g. Antares ( $\alpha$ Sco, M1 Ib + B2.5 V), the blue companion moving through the outer RSG wind produces a photoionized cavity and a wake (Braun et al., 2012). In principle, one might speculate that X-rays are generated either in the wind-wind collision zone or in the photoionization wake. However, Antares is X-ray dark.

The enigmatic L-type supergiant V838 Mon may be a merger product between a massive B-type star and an intermediate mass star (Tylenda and Soker, 2006). It is theoretically expected that merger products may have strong magnetic fields (Tutukov and Fedorova, 2010). Therefore, the merger hypothesis could be potentially tested by X-ray observations of the post-merger object. The post outburst observations of V838 Mon did not reveal X-ray emission. However, later on a new X-ray source was found a few arcsec away from V838 Mon. One of the possible interpretations of this X-ray source is the interaction between the V838Mon ejecta and the B3 V companion (Antonini et al., 2010).
Interestingly, it was recently suggested that a merger event triggered the the great eruption of $\eta$ Carinae (Portegies Zwart and van den Heuvel, 2016). This LBV is one of the most massive stars in the Galaxy and a prodigious X-ray source (Seward et al., 1979). The X-rays in $\eta$ Car are produced in a collision of the LBV wind with the wind of its early-type companion (e.g. Damineli et al., 2000; Corcoran et al., 2001; Hamaguchi et al., 2007; Parkin et al., 2009). As in other colliding wind systems, X-rays provide important information about orbital, stellar, and wind parameters (see review by Rauw \& Nazé in this volume).

Another X-ray bright LBV star is HD 5980 in the Small Magellanic Cloud. The X-ray emission from this system is also powered by the collision between stellar winds in a multiple system (Nazé et al., 2007). In general, it seems that all LBV stars detected in Xrays are multiple systems, where collisions between stellar winds are taking place (Oskinova, 2005; Nazé et al., 2012). The lack of $\mathrm{X}$-ray emission from single LBVs is expected because their winds are quite slow, with velocities $\sim 100 \mathrm{~km} \mathrm{~s}^{-1}$, and very dense, with $\dot{M}$ as high as $10^{-3} M_{\odot} \mathrm{yr}^{-1}$ (Hillier et al., 2001; Groh et al., 2009; Maryeva and Abolmasov, 2012). At such low wind velocities wind shocks (even if present) cannot produce sufficiently hot plasma. Moreover, any Xrays produced in the wind or at the wind base would be fully absorbed (e.g. Oskinova, 2005).

WR spectra are divided into three broad spectroscopic classes, WN, WC, and WO. WN stars display CNO-processed matter in 
a strong stellar wind. The cooler, late WN subtypes (WNL) usually contain some rest of hydrogen in their atmospheres, while the hotter, early subtypes (WNE) are hydrogen free (Hamann et al., 1991). The WNL evolutionary stage can precede the LBV stage (Langer et al., 1994; Langer, 2012). Typically, WNL stars are significantly more luminous than WNE stars (Hamann et al., 2006). The WN phase may be followed by the WC and WO stages, when the products of helium burning appear in the stellar atmosphere (see Sander et al., 2012; Tramper et al., 2015, for recent analyses).

Already from the analysis of early Einstein $\mathrm{X}$-ray telescope observations it was found that binary WR stars are usually bright Xrays sources, while single $\mathrm{WR}$ stars are relatively faint (Pollock, 1987).

In binary WR stars the bulk of X-rays is produced in colliding winds. The WN, WC, and WO type colliding wind binaries are all bright X-ray sources that usually show variability explained by orbital motion (e.g. Pollock et al., 2005; Sugawara et al., 2008; Lomax et al., 2015; Zhekov, 2012). In some objects, besides X-rays, non-thermal radio emission (De Becker and Raucq, 2013) and dust are produced (Williams et al., 1990; Tuthill et al., 1999).

The X-ray spectra of WR stars are well reproduced by multi-temperature thermal plasma models with temperatures between $1 \mathrm{MK}$ up to $50 \mathrm{MK}$ (Skinner et al., 2002, 2010; Ignace et al., 2003; Oskinova et al., 2012). The X-ray production mechanisms in these stars are not yet understood, but it appears that $\mathrm{X}$-ray production, like in $\mathrm{O}$ type stars, is an intrinsic ingredient of the stellar wind driving mechanism (see K. Gayley, this volume).

Yet there are significant differences in the $\mathrm{X}$-ray properties of different types of WR stars. The WNE stars have fast stellar winds, similar effective temperatures and luminosities (Hamann et al., 2006). All well observed single WN stars are X-ray sources with $L_{\mathrm{X}} \approx 2 \ldots 6 \times 10^{32} \mathrm{erg} \mathrm{s}^{-1}$ and an emission measure weighted average temperatures of $\langle T\rangle \approx 5 \mathrm{MK}$. Chené et al. (2011); Chené and St-Louis (2011) investigated the large-scale spectroscopic and photometric optical variability in apparently single WR stars. Such variability was found in four WN stars (WR1, WR6, WR110, WR 134), and attributed to the presence of CIRs. All these stars have similar X-ray properties. Chené et al. (2011) suggested as an explanation for these quite hard X-rays the shocks which are associated with the CIRs.

So far, a high-resolution X-ray spectrum is available for only one single WR star - EZ CMa (WR 6) of spectral type WR4 (Oskinova et al., 2012; Huenemoerder et al., 2015). Figure 1 shows its RGS spectrum. The resolved X-ray lines are broad and strongly blueshifted as expected when the radiation forms in the outer wind and suffers strong continuum absorption (Macfarlane et al., 1991; Ignace, 2001). The analysis of line ratios in Helike ions confirms that the radiation is formed far above the photosphere. Even the hottest plasma is seen at more than ten stellar radii. The fluorescent Fe $\mathrm{K} \alpha$ line is seen in the $\mathrm{X}$ ray spectrum. This shows that dense and cool matter is illuminated by the nearby $\mathrm{X}$ - 
ray sources.

X-ray spectroscopy provides an excellent probe of metal abundances in stellar winds. Some of these abundances could not be measured at other wavelengths. E.g., sodium lines were detected in the Chandra spectra of WR 6. The spectral analysis revealed that the sodium abundance is enhanced in this star in accordance with its evolutionary state (Huenemoerder et al., 2015).

The WNL stars are rather weak X-ray sources with X-ray luminosities not exceeding $L_{\mathrm{X}} \approx 10^{32} \mathrm{erg} \mathrm{s}^{-1}$ (Wrigge et al., 1994; Pollock et al., 1995; Ignace et al., 2000; Oskinova, 2005). Skinner et al. (2012) reported a $2 \sigma$ detection of the WN8h star WR16 with $L_{\mathrm{X}} \approx 10^{31} \mathrm{erg} \mathrm{s}^{-1}$. Some WNL stars were not detected in X-rays despite quite sensitive observations, e.g. Gosset et al. (2005) found an upper limit to the X-ray luminosity of WR40 (WN8h) of $L_{\mathrm{X}}<4 \times 10^{31} \mathrm{erg} \mathrm{s}^{-1}$. This is especially puzzling, because the wind of WR40 is more transparent for $\mathrm{X}$-rays than the wind of WR6 (WN4), yet the latter is > 100 times more X-ray luminous than the former.

The WC stars are X-ray faint with $L_{\mathrm{X}} / L_{\text {bol }}<10^{-9}$, likely because of the high opacity of their dense and strongly metal enriched winds (Oskinova et al., 2003). This conjecture is further supported by the first detection of faint X-ray emission from a single WC star. Rauw et al. (2015b) report the detection of WR144 with $L_{\mathrm{X}} \approx 10^{30} \mathrm{erg} \mathrm{s}^{-1}$. With its spectral type WC4, WR144 is one of the hottest WC stars with the fastest wind among this class (Sander et al., 2012). Thus its wind significantly less opaque for X-rays than in cooler WC types. The intrinsic X- ray faintness of single WC stars provides an excellent diagnostic tool of binarity: X-ray bright WC stars must be colliding wind systems (e.g. Clark et al., 2008; Oskinova and Hamann, 2008; Hyodo et al., 2008; Mauerhan et al., 2011; Nebot Gómez-Morán et al., 2015).

In WO winds the absorption is significantly reduced compared to other WR stars, owing to lower wind column densities. Moreover, the very high stellar effective temperature of WO stars results in a higher degree of wind ionization compared to $\mathrm{WN}$ and WC stars, farther reducing the opacity for X-rays (Oskinova et al., 2012). The wind speed in WO stars is very high, up to $6000 \mathrm{~km} \mathrm{~s}^{-1}$ (Sander et al., 2012; Tramper et al., 2015). Hence, if such stellar wind is shocked, the temperature of the heated plasma can reach up to $100 \mathrm{MK}$. The resulting radiation would be rather hard and would easier escape from the stellar wind. Thus, one may expect that WO stars are detectable X-ray sources. These predictions were confirmed by the XMM-Newton and Chandra detections of hard X-ray radiation from the WO2 star WR 142 with $L_{\mathrm{X}} \approx 10^{31} \mathrm{erg} \mathrm{s}^{-1}$ and $L_{\mathrm{X}} / L_{\mathrm{bol}} \sim 10^{-8}$ (Oskinova et al., 2009; Sokal et al., 2010).

Whatever is the mechanism of X-ray production in WR stars, the observed X-ray emission allows to probe their strong stellar winds. With the notable exception of WN8h type stars, the picture is emerging where the X-ray properties of WN, WC, and WO stars are chiefly determined by their wind opacity. The wind opacity of WC stars is higher than of WN and WO stars. As a result, we are 
able to see X-rays emerging from the winds of WN and WO stars but not from WC type stars.

\section{Summary}

Observations in the X-ray band of the electromagnetic spectrum provide an important diagnostic tool for stellar winds. In early Btype and late O-type dwarfs, the bulk of stellar wind may be in a hot phase, making the observations at X-ray wavelengths, the primary tool to study these most numerous massive stars.

In $\mathrm{OB}$ giants and supergiants, the wind is mainly in the cool phase and quite opaque for the X-rays. Since the stellar winds are clumped, X-rays emitted from embedded sources can escape and reach the observer. The high-resolution X-ray spectra of OB supergiants allow detailed studies of $\mathrm{X}$ ray transfer in their winds. A brief parameter study shows that the fitting of X-ray line profiles by means of a simple model does not provide a reliable tool to measure stellar massloss rates. A multiwavelength spectroscopic analysis, when the X-ray data are analyzed consistently with optical and UV spectra, is required to derive realistic stellar and wind parameters.

X-ray variability emerges as a new powerful tool to understand stellar wind structures. Pronounced stochastic X-ray variability has not been detected from stellar winds so far. However, all stars that were observed with sufficiently long exposures show X-ray variability on time scales of days, similar to the variability in $\mathrm{H} \alpha$ and $\mathrm{UV}$ lines. A likely mechanism for this variability is by corotating interaction regions.

Massive stars at the final stages of their evolution have diverse X-ray properties. The RSG stars appear to be X-ray dark. Single LBV stars and late sub-type WC stars are not observable as X-ray sources, in agreement with theoretical expectations. Single WN stars emit X-rays, albeit the responsible mechanism is not yet fully understood. The hottest among all massive stars, the early sub-type WC and WO stars, are found to be $\mathrm{X}$-ray sources, likely indicating that in these extreme objects the attenuation of $\mathrm{X}$-ray is sufficiently low.

The author is indebted to both anonymous referees who provided important and detailed comments leading to a significant improvement of the manuscript. Support from DLR grant 50 OR 1302 is acknowledged.

\section{References}

Abbott, D.C., 1979. The domain of radiatively driven mass loss in the H-R diagram, in: Conti, P.S., De Loore, C.W.H. (Eds.), Mass Loss and Evolution of O-Type Stars, pp. 237-239.

Abbott, M.J., Friend, D.B., 1989. A radiation-driven stellar wind model with a line force cutoff. ApJ 345, 505-510. doi:10.1086/167925.

Agrawal, P.C., Singh, K.P., Riegler, G.R., Stern, R.A., 1984. An X-ray survey of beta Cephei stars. MNRAS 208, 845-851. 
Alecian, E., Kochukhov, O., Neiner, C., Wade, G.A., de Batz, B., Henrichs, H., Grunhut, J.H., Bouret, J.C., Briquet, M., Gagne, M., Naze, Y., Oksala, M.E., Rivinius, T., Townsend, R.H.D., Walborn, N.R., Weiss, W., Mimes Collaboration, 2011. First HARPSpol discoveries of magnetic fields in massive stars. A\&A 536, L6. doi:10.1051/0004-6361/201118354, arXiv:1111. 3433.

Antonini, F., Montez, Jr., R., Kastner, J.H., Bond, H.E., Soker, N., Tylenda, R., Starrfield, S., Behar, E., 2010. XMM-Newton Detection of a Transient X-ray Source in the Vicinity of V838 Monocerotis. ApJ 717, 795802. doi:10.1088/0004-637X/717/2/795, arXiv:0910.0503.

Babel, J., 1996. The fading of radiatively driven winds in B stars. A\&A 309, 867878.

Babel, J., Montmerle, T., 1997. X-ray emission from Ap-Bp stars: a magnetically confined wind-shock model for IQ Aur. A\&A 323, 121-138.

Berghoefer, T.W., Baade, D., Schmitt, J.H.M.M., Kudritzki, R.P., Puls, J., Hillier, D.J., Pauldrach, A.W.A., 1996. Correlated variability in the X-ray and $\mathrm{H} \alpha$ emission from the O4If supergiant $\zeta$ Puppis. A\&A 306, $899-912$.

Berghoefer, T.W., Schmitt, J.H.M.M., Danner, R., Cassinelli, J.P., 1997. X-ray properties of bright OB-type stars detected in the ROSAT all-sky survey. A\&A 322, 167174 .

Berghofer, T.W., Schmitt, J.H.M.M., 1994. X-ray Variability in the Hot Supergiant zeta Orionis. Science 265, 1689-1691. doi:10.1126/science.265.5179.1689.

Blumenthal, G.R., Drake, G.W.F., Tucker, W.H., 1972. Ratio of Line Intensities in Helium-Like Ions as a Density Indicator. ApJ 172, 205-212. doi:10.1086/151340.

Bouret, J.C., Donati, J.F., Martins, F., Escolano, C., Marcolino, W., Lanz, T., Howarth, I.D., 2008. The weak magnetic field of the O9.7 supergiant $\zeta$ OrionisA. MNRAS 389, 75-85. doi:10.1111/j.1365-2966.2008.13575.x, arXiv:0806.2162.

Bouret, J.C., Lanz, T., Hillier, D.J., 2005. Lower mass loss rates in O-type stars: Spectral signatures of dense clumps in the wind of two Galactic O4 stars. A\&A 438, 301-316. doi:10.1051/0004-6361:20042531, arXiv: astro-ph/0412346.

Bozzo, E., Giunta, A., Cusumano, G., Ferrigno, C., Walter, R., Campana, S., Falanga, M., Israel, G., Stella, L., 2011. XMM-Newton observations of IGR J18410-0535: the ingestion of a clump by a supergiant fast X-ray transient. A\&A 531, A130. doi:10.1051/0004-6361/201116726, arXiv:1106.5125. 
Braun, K., Baade, R., Reimers, D., Hagen, H.J., 2012. A hydrodynamic study of the circumstellar envelope of ¡ASTROBJ $\_\alpha$ Scorpii/ASTROBJ . $_{\text {A }}$ A\&A 546, A3. doi:10.1051/0004-6361/201219659, arXiv: 1208.5866.

Brinkman, A.C., Gunsing, C.J.T., Kaastra, J.S., van der Meer, R.L.J., Mewe, R., Paerels, F., Raassen, A.J.J., van Rooijen, J.J., Bräuninger, H., Burkert, W., Burwitz, V., Hartner, G., Predehl, P., Ness, J.U., Schmitt, J.H.M.M., Drake, J.J., Johnson, O., Juda, M., Kashyap, V., Murray, S.S., Pease, D., Ratzlaff, P., Wargelin, B.J., 2000. First Light Measurements of Capella with the Low-Energy Transmission Grating Spectrometer aboard the Chandra X-Ray Observatory. ApJ 530, L111-L114. doi:10.1086/312504, arXiv:astro-ph/0001034.

Canizares, C.R., Davis, J.E., Dewey, D., Flanagan, K.A., Galton, E.B., Huenemoerder, D.P., Ishibashi, K., Markert, T.H., Marshall, H.L., McGuirk, M., Schattenburg, M.L., Schulz, N.S., Smith, H.I., Wise, M., 2005. The Chandra High-Energy Transmission Grating: Design, Fabrication, Ground Calibration, and 5 Years in Flight. PASP 117, 1144-1171. doi:10.1086/432898, arXiv:astro-ph/0507035.

Cantiello, M., Braithwaite, J., 2011. Magnetic spots on hot massive stars. A\&A 534, A140. doi:10.1051/0004-6361/201117512, arXiv:1108.2030.
Carneiro, L.P., Puls, J., Sundqvist, J.O., Hoffmann, T.L., 2016. Atmospheric NLTEModels for the Spectroscopic Analysis of Blue Stars with Winds. III. X-ray emission from wind-embedded shocks. ArXiv e-prints arXiv: 1603.01177.

Cassinelli, J.P., Cohen, D.H., Macfarlane, J.J., Drew, J.E., Lynas-Gray, A.E., Hubeny, I., Vallerga, J.V., Welsh, B.Y., Hoare, M.G., 1996. EUVE Spectroscopy of $\beta$ Canis Majoris (B1 II-III) from 500 Angstrom to 700 Angstrom. ApJ 460, 949 - 963. doi:10.1086/177023.

Cassinelli, J.P., Cohen, D.H., Macfarlane, J.J., Sanders, W.T., Welsh, B.Y., 1994. X-ray emission from near-mainsequence B stars. ApJ 421, 705-717. doi:10.1086/173683.

Cassinelli, J.P., Ignace, R., Waldron, W.L., Cho, J., Murphy, N.A., Lazarian, A., 2008. The Effects of Clumps in Explaining X-Ray Emission Lines from Hot Stars. ApJ 683, 1052-1062. doi:10.1086/589760, arXiv:0804.4680.

Cassinelli, J.P., Miller, N.A., Waldron, W.L., MacFarlane, J.J., Cohen, D.H., 2001. Chandra Detection of Dopplershifted X-Ray Line Profiles from the Wind of $\zeta$ Puppis (O4 F). ApJ 554, L55-L58. doi:10.1086/320916, arXiv:astro-ph/0104107.

Cassinelli, J.P., Olson, G.L., 1979. The effects of coronal regions on the X-ray flux and ionization conditions in the winds of 
OB supergiants and Of stars. ApJ 229, 304-317. doi:10.1086/156956.

Cassinelli, J.P., Swank, J.H., 1983. X-ray spectra of Orion OB supergiants. ApJ 271, 681-690. doi:10.1086/161235.

Cassinelli, J.P., Waldron, W.L., Sanders, W.T., Harnden, Jr., F.R., Rosner, R., Vaiana, G.S., 1981. X-ray emission from Of stars and OB supergiants. ApJ 250, 677686. doi:10.1086/159414.

Castor, J.I., Abbott, D.C., Klein, R.I., 1975. Radiation-driven winds in Of stars. ApJ 195, 157-174. doi:10.1086/153315.

Castro, N., Fossati, L., Hubrig, S., SimónDíaz, S., Schöller, M., Ilyin, I., Carrol, T.A., Langer, N., Morel, T., Schneider, F.R.N., Przybilla, N., Herrero, A., de Koter, A., Oskinova, L.M., Reisenegger, A., Sana, H., BOB Collaboration, 2015. B fields in OB stars (BOB). Detection of a strong magnetic field in the O9.7 V star HD 54879. A\&A 581, A81. doi:10.1051/0004-6361/201425354, arXiv: 1507.03591.

Chené, A.N., Moffat, A.F.J., Cameron, C., Fahed, R., Gamen, R.C., Lefèvre, L., Rowe, J.F., St-louis, N., Muntean, V., De La Chevrotière, A., Guenther, D.B., Kuschnig, R., Matthews, J.M., Rucinski, S.M., Sasselov, D., Weiss, W.W., 2011. WR 110: A Single Wolf-Rayet Star with Corotating Interaction Regions in its Wind? ApJ 735, 3443. doi:10.1088/0004-637X/735/1/34, arXiv: 1105.0919.
Chené, A.N., St-Louis, N., 2011. A Systematic Search for Corotating Interaction Regions in Apparently Single Galactic Wolf-Rayet Stars. II. A Global View of the Wind Variability. ApJ 736, 140156. doi:10.1088/0004-637X/736/2/140, arXiv:1105.5133.

Chlebowski, T., Garmany, C.D., 1991. On winds and X-rays of O-type stars. ApJ 368, 241-251. doi:10.1086/169687.

Clark, J.S., Muno, M.P., Negueruela, I., Dougherty, S.M., Crowther, P.A., Goodwin, S.P., de Grijs, R., 2008. Unveiling the $\mathrm{X}$-ray point source population of the Young Massive Cluster Westerlund 1. A\&A 477, 147-163. doi:10.1051/0004-6361:20077186.

Cohen, D.H., de Messières, G.E., MacFarlane, J.J., Miller, N.A., Cassinelli, J.P., Owocki, S.P., Liedahl, D.A., 2003. High-Resolution Chandra Spectroscopy of $\tau$ Scorpii: A Narrow-Line X-Ray Spectrum from a Hot Star. ApJ 586, 495-505. doi:10.1086/367553, arXiv: astro-ph/0211412.

Cohen, D.H., Gagné, M., Leutenegger, M.A., MacArthur, J.P., Wollman, E.E., Sundqvist, J.O., Fullerton, A.W., Owocki, S.P., 2011. Chandra X-ray spectroscopy of the very early O supergiant HD 93129A: constraints on wind shocks and the mass-loss rate. MNRAS 415, 3354-3364. doi:10.1111/j.1365-2966.2011.18952.x, arXiv:1104.4786. 
Cohen, D.H., Leutenegger, M.A., Grizzard, K.T., Reed, C.L., Kramer, R.H., Owocki, S.P., 2006. Wind signatures in the X-ray emission-line profiles of the late-O supergiant $\zeta$ Orionis. MNRAS 368, 1905-1916. doi:10.1111/j.1365-2966.2006.10259.x, arXiv: astro-ph/0602599.

Cohen, D.H., Leutenegger, M.A., Wollman, E.E., Zsargó, J., Hillier, D.J., Townsend, R.H.D., Owocki, S.P., 2010. A mass-loss rate determination for $\zeta$ Puppis from the quantitative analysis of X-ray emissionline profiles. MNRAS 405, 2391-2405. doi:10.1111/j.1365-2966.2010.16606.x, arXiv: 1003.0892.

Cohen, D.H., Wollman, E.E., Leutenegger, M.A., Sundqvist, J.O., Fullerton, A.W., Zsargó, J., Owocki, S.P., 2014. Measuring mass-loss rates and constraining shock physics using $\mathrm{X}$-ray line profiles of $\mathrm{O}$ stars from the Chandra archive. MNRAS 439, 908-923. doi:10.1093/mnras/stu008, arXiv:1401.7995.

Corcoran, M.F., Ishibashi, K., Swank, J.H., Petre, R., 2001. The X-Ray Light Curve of $\eta$ Carinae: Refinement of the Orbit and Evidence for Phase-dependent Mass Loss. ApJ 547, 1034-1039. doi:10.1086/318416.

Corcoran, M.F., Nichols, J.S., Pablo, H., Shenar, T., Pollock, A.M.T., Waldron, W.L., Moffat, A.F.J., Richardson, N.D., Russell, C.M.P., Hamaguchi, K., Huenemoerder, D.P., Oskinova, L., Hamann, W.R., Nazé, Y., Ignace, R., Evans, N.R., Lomax, J.R., Hoffman, J.L., Gayley, K.,
Owocki, S.P., Leutenegger, M., Gull, T.R., Hole, K.T., Lauer, J., Iping, R.C., 2015. A Coordinated X-Ray and Optical Campaign of the Nearest Massive Eclipsing Binary, $\delta$ Orionis Aa. I. Overview of the X-Ray Spectrum. ApJ 809, 132. doi:10.1088/0004-637X/809/2/132, arXiv: 1507.05101.

Cranmer, S.R., Owocki, S.P., 1996. Hydrodynamical Simulations of Corotating Interaction Regions and Discrete Absorption Components in Rotating O-Star Winds. ApJ 462, 469-488. doi:10.1086/177166, arXiv: astro-ph/9508004.

Damineli, A., Kaufer, A., Wolf, B., Stahl, O., Lopes, D.F., de Araújo, F.X., 2000. $\eta$ Carinae: Binarity Confirmed. ApJ 528, L101-L104. doi:10.1086/312441, arXiv: astro-ph/9912387.

De Becker, M., Raucq, F., 2013. Catalogue of particle-accelerating collidingwind binaries. A\&A 558, A28. doi:10.1051/0004-6361/201322074, arXiv: 1308.3149 .

den Herder, J.W., Brinkman, A.C., Kahn, S.M., Branduardi-Raymont, G., Thomsen, K., Aarts, H., Audard, M., Bixler, J.V., den Boggende, A.J., Cottam, J., Decker, T., Dubbeldam, L., Erd, C., Goulooze, H., Güdel, M., Guttridge, P., Hailey, C.J., Janabi, K.A., Kaastra, J.S., de Korte, P.A.J., van Leeuwen, B.J., Mauche, C., McCalden, A.J., Mewe, R., Naber, A., Paerels, F.B., Peterson, J.R., Rasmussen, A.P., Rees, K., Sakelliou, I., Sako, M., Spodek, J., 
Stern, M., Tamura, T., Tandy, J., de Vries, C.P., Welch, S., Zehnder, A., 2001. The Reflection Grating Spectrometer on board XMM-Newton. A\&A 365, L7-L17. doi:10.1051/0004-6361:20000058.

Donati, J.F., Howarth, I.D., Jardine, M.M., Petit, P., Catala, C., Landstreet, J.D., Bouret, J.C., Alecian, E., Barnes, J.R., Forveille, T., Paletou, F., Manset, N., 2006. The surprising magnetic topology of $\tau$ Sco: fossil remnant or dynamo output? MNRAS 370, 629-644. doi:10.1111/j.1365-2966.2006.10558.x, arXiv:astro-ph/0606156.

Donati, J.F., Wade, G.A., Babel, J., Henrichs, H.f., de Jong, J.A., Harries, T.J., 2001. The magnetic field and wind confinement of $\beta$ Cephei: new clues for interpreting the Be phenomenon? MNRAS 326, 1265-1278. doi:10.1111/j.1365-8711.2001.04713.x.

Drew, J.E., Hoare, M.G., Denby, M., 1994. Soft X-ray observations of the early B giants Beta and Epsilon CMa. MNRAS 266, 917-924.

Dziembowski, W.A., Pamiatnykh, A.A., 1993. The opacity mechanism in B-type stars. I - Unstable modes in Beta Cephei star models. MNRAS 262, 204-212.

Engle, S.G., Guinan, E.F., Harper, G.M., Neilson, H.R., Remage Evans, N., 2014. The Secret Lives of Cepheids: Evolutionary Changes and Pulsationinduced Shock Heating in the Prototype
Classical Cepheid $\delta$ Cep. ApJ 794, 80. doi:10.1088/0004-637X/794/1/80, arXiv: 1409.8628.

Evans, N.R., DeGioia-Eastwood, K., Gagné, M., Townsley, L., Broos, P., Wolk, S. Nazé, Y., Corcoran, M., Oskinova, L., Moffat, A.F.J., Wang, J., Walborn, N.R., 2011. The Search for Low-mass Companions of B Stars in the Carina Nebula Cluster Trumpler 16. ApJS 194, 13. doi:10.1088/0067-0049/194/1/13, arXiv: 1103.1344.

Eversberg, T., Lépine, S., Moffat, A.F.J., 1998. Outmoving Clumps in the Wind of the Hot O Supergiant $\zeta$ Puppis. ApJ 494, 799-805. doi:10.1086/305218.

Favata, F., Neiner, C., Testa, P., Hussain, G., Sanz-Forcada, J., $2009 . \quad$ Testing magnetically confined wind shock models for $\beta$ Cephei using XMMNewton and Chandra phase-resolved X-ray observations. A\&A 495, 217 229. doi:10.1051/0004-6361:20078529, arXiv:0806.2275.

Feldmeier, A., Kudritzki, R.P., Palsa, R., Pauldrach, A.W.A., Puls, J., 1997a. The $\mathrm{X}$-ray emission from shock cooling zones in O star winds. A\&A 320, 899-912.

Feldmeier, A., Oskinova, L., Hamann, W.R., 2003. X-ray line emission from a fragmented stellar wind. A\&A 403, 217224. doi:10.1051/0004-6361:20030231, arXiv:astro-ph/0302516. 
Feldmeier, A., Puls, J., Pauldrach, A.W.A., 1997b. A possible origin for X-rays from O stars. A\&A 322, 878-895.

Fossati, L., Castro, N., Morel, T., Langer, N., Briquet, M., Carroll, T.A., Hubrig, S., Nieva, M.F., Oskinova, L.M., Przybilla, N., Schneider, F.R.N., Schöller, M., SimónDíaz, S., Ilyin, I., de Koter, A., Reisenegger, A., Sana, H., 2015. B fields in OB stars (BOB): on the detection of weak magnetic fields in the two early B-type stars $\beta$ CMa and $\epsilon$ CMa. Possible lack of a "magnetic desert" in massive stars. A\&A 574, A20. doi:10.1051/0004-6361/201424986, arXiv:1411.6490.

Fullerton, A.W., Massa, D.L., Prinja, R.K., 2006. The Discordance of Mass-Loss Estimates for Galactic O-Type Stars. ApJ 637, 1025-1039. doi:10.1086/498560, arXiv: astro-ph/0510252.

Giménez-García, A., Torrejón, J.M., Eikmann, W., Martínez-Núñez, S., Oskinova, L.M., Rodes-Roca, J.J., Bernabéu, G., 2015. An XMM-Newton view of $\mathrm{FeK} \alpha$ in high-mass X-ray binaries. A\&A 576, A108. doi:10.1051/0004-6361/201425004.

Gosset, E., Nazé, Y., Claeskens, J.F., Rauw, G., Vreux, J.M., Sana, H., 2005. An XMM-Newton look at the Wolf-Rayet star WR 40. The star itself, its nebula and its neighbours. A\&A 429, 685-704. doi:10.1051/0004-6361:20040286.

Groh, J.H., Hillier, D.J., Damineli, A., Whitelock, P.A., Marang, F., Rossi, C.,
2009. On the Nature of the Prototype Luminous Blue Variable Ag Carinae. I. Fundamental Parameters During Visual Minimum Phases and Changes in the Bolometric Luminosity During the S-Dor Cycle. ApJ 698, 1698-1720. doi:10.1088/0004-637X/698/2/1698, arXiv:0904.2363.

Grunhut, J.H., Wade, G.A., Marcolino, W.L.F., Petit, V., Henrichs, H.F., Cohen, D.H., Alecian, E., Bohlender, D., Bouret, J.C., Kochukhov, O., Neiner, C., St-Louis, N., Townsend, R.H.D., 2009. Discovery of a magnetic field in the O9 sub-giant star HD 57682 by the MiMeS Collaboration. MNRAS 400, L94-L98. doi:10.1111/j.1745-3933.2009.00771.x, arXiv:0910.0214.

Gullikson, K., Dodson-Robinson, S., 2013. Detection of Low-Mass-ratio Stellar Binary Systems. AJ 145, 3. doi:10.1088/0004-6256/145/1/3, arXiv: 1210.6360 .

Guo, J.H., 2010. X-ray temperatures and their radial distributions from winds of O-type supergiants: the effects of clumps. A\&A 512, A50. doi:10.1051/0004-6361/200912872.

Hamaguchi, K., Corcoran, M.F., Gull, T., Ishibashi, K., Pittard, J.M., Hillier, D.J., Damineli, A., Davidson, K., Nielsen, K.E., Kober, G.V., 2007. XRay Spectral Variation of $\eta$ Carinae through the 2003 X-Ray Minimum. ApJ 
663, 522-542. doi:10.1086/518101, arXiv: astro-ph/0702409.

Hamann, W.R., 1981. The expanding envelope of Tau Scorpii - A detailed UV-line fit. A\&A 100, 169-174.

Hamann, W.R., Duennebeil, G., Koesterke, L., Wessolowski, U., Schmutz, W., 1991. Spectral analyses of Wolf-Rayet stars - Hydrogen abundances in WN subtypes. A\&A 249, 443-454.

Hamann, W.R., Gräfener, G., Liermann, A., 2006. The Galactic WN stars. Spectral analyses with lineblanketed model atmospheres versus stellar evolution models with and without rotation. A\&A 457, 1015-1031. doi:10.1051/0004-6361:20065052, arXiv: astro-ph/0608078.

Hamann, W.R., Koesterke, L., 1998. Spectrum formation in clumped stellar winds: consequences for the analyses of WolfRayet spectra. A\&A 335, 1003-1008.

Hamann, W.R., Oskinova, L.M., Feldmeier, A., 2008. Spectrum formation in clumpy stellar winds, in: Hamann, W.R., Feldmeier, A., Oskinova, L.M. (Eds.), Clumping in Hot-Star Winds: proceedings of an international workshop held in Potsdam, Germany, 18. - 22. June 2007, Universitätsverlag Potsdam., p. 75.

Harnden, Jr., F.R., Branduardi, G., Gorenstein, P., Grindlay, J., Rosner, R., Topka, K., Elvis, M., Pye, J.P., Vaiana, G.S., 1979. Discovery of an X-ray star association in
VI Cygni /Cyg OB2/. ApJ 234, L51-L54. doi:10.1086/183107.

Hervé, A., Rauw, G., Nazé, Y., 2013. A detailed X-ray investigation of $\zeta$ Puppis. III. Spectral analysis of the whole RGS spectrum. A\&A 551, A83. doi:10.1051/0004-6361/201219734, arXiv: 1301.5090.

Hervé, A., Rauw, G., Nazé, Y., Foster, A., 2012. Global Modeling of X-Ray Spectra Produced in O-type Star Winds. ApJ 748, 89. doi:10.1088/0004-637X/748/2/89, arXiv: 1201.4716 .

Hillier, D.J., 1991. The effects of electron scattering and wind clumping for early emission line stars. A\&A 247, 455-468.

Hillier, D.J., Davidson, K., Ishibashi, K., Gull, T., 2001. On the Nature of the Central Source in $\eta$ Carinae. ApJ 553, 837-860. doi:10. 1086/320948.

Hillier, D.J., Kudritzki, R.P., Pauldrach, A.W., Baade, D., Cassinelli, J.P., Puls, J., Schmitt, J.H.M.M., 1993. The 0.1-2.5KEV X-Ray Spectrum of the O4F-STAR Zeta-Puppis. A\&A 276, 117-128.

Howk, J.C., Cassinelli, J.P., Bjorkman, J.E., Lamers, H.J.G.L.M., 2000. Stagnation and Infall of Dense Clumps in the Stellar Wind of $\tau$ Scorpii. ApJ 534, 348-358. doi:10.1086/308730, arXiv: astro-ph/9912360.

Hubrig, S., Briquet, M., Morel, T., Schöller, M., González, J.F., De Cat, P., 2008. 
New insights into the nature of the peculiar star $\theta$ Carinae. A\&A 488, 287296. doi:10.1051/0004-6361:200809972, arXiv:0807. 2067.

Hubrig, S., Briquet, M., Schöller, M., De Cat, P., Mathys, G., Aerts, C., 2006. Discovery of magnetic fields in the $\beta$ Cephei star $\xi^{1} \mathrm{CMa}$ and in several slowly pulsating B stars*. MNRAS 369, L61-L65. doi:10.1111/j.1745-3933.2006.00175.x, arXiv:astro-ph/0604283.

Hubrig, S., Oskinova, L.M., Schöller, M., 2011. First detection of a magnetic field in the fast rotating runaway Oe star $\zeta$ Ophiuchi. Astronomische Nachrichten 332, 147-152. doi:10.1002/asna.201111516, arXiv: 1101.5500.

Huenemoerder, D.P., Gayley, K.G., Hamann, W.R., Ignace, R., Nichols, J.S., Oskinova, L., Pollock, A.M.T., Schulz, N.S., Shenar, T., 2015. Probing Wolf-Rayet Winds: Chandra/HETG XRay Spectra of WR 6. ApJ 815, 29. doi:10.1088/0004-637X/815/1/29, arXiv: 1511.01832.

Huenemoerder, D.P., Oskinova, L.M., Ignace, R., Waldron, W.L., Todt, H., Hamaguchi, K., Kitamoto, S., 2012. On the Weak-wind Problem in Massive Stars: X-Ray Spectra Reveal a Massive Hot Wind in $\mu$ Columbae. ApJ 756, L34. doi:10 . 1088/2041-8205/756/2/L34, arXiv: 1208.0820.

Hyodo, Y., Tsujimoto, M., Koyama, K., Nishiyama, S., Nagata, T., Sakon, I., Mu- rakami, H., Matsumoto, H., 2008. Suzaku X-Ray Spectroscopy of a Peculiar Hot Star in the Galactic Center Region. PASJ 60, 173-182. arXiv:0712.0280.

Ignace, R., 2001. Theoretical Profile Shapes for Optically Thin X-Ray Emission Lines from Spherical Stellar Winds. ApJ 549, L119-L123. doi:10.1086/319141.

Ignace, R., 2016. Long-wavelength, freefree spectral energy distributions from porous stellar winds. MNRAS 457, 4123-4134. doi:10.1093/mnras/stw216, arXiv: 1601.06715.

Ignace, R., Gayley, K.G., 2002. Profile Shapes for Optically Thick X-Ray Emission Lines from Stellar Winds. ApJ 568, 954-964. doi:10.1086/339059.

Ignace, R., Gayley, K.G., Hamann, W.R., Huenemoerder, D.P., Oskinova, L.M., Pollock, A.M.T., McFall, M., 2013a. The XMM-Newton/EPIC X-Ray Light Curve Analysis of WR 6. ApJ 775, 29. doi:10.1088/0004-637X/775/1/29, arXiv: 1307.7074 .

Ignace, R., Oskinova, L.M., 1999. An explanation of observed trends in the Xray emission from single Wolf-Rayet stars. A\&A 348, L45-L48.

Ignace, R., Oskinova, L.M., Brown, J.C., 2003. XMM-Newton observations of the nitrogen-rich Wolf-Rayet star WR 1. A\&A 408, 353-361. doi:10.1051/0004-6361:20031024. 
Ignace, R., Oskinova, L.M., Foullon, C., 2000. Exospheric models for the X-ray emission from single WolfRayet stars. MNRAS 318, 214-226. doi:10.1046/j.1365-8711.2000.03744.x, arXiv:astro-ph/0111533.

Ignace, R., Oskinova, L.M., Jardine, M., Cassinelli, J.P., Cohen, D.H., Donati, J.F., Townsend, R.H.D., ud-Doula, A., 2010. A Multiphase Suzaku Study of X-rays from $\tau$ Sco. ApJ 721, 1412-1420. doi:10.1088/0004-637X/721/2/1412, arXiv:1008.1552.

Ignace, R., Oskinova, L.M., Massa, D., 2013b. A report on the X-ray properties of the $\tau$ Sco-like stars. MNRAS 429, 516-522. doi:10.1093/mnras/sts358, arXiv: 1211.0861.

Ignace, R., Waldron, W.L., Cassinelli, J.P., Burke, A.E., 2012. X-Ray Emission Line Profiles from Wind Clump Bow Shocks in Massive Stars. ApJ 750, 40. doi:10.1088/0004-637X/750/1/40, arXiv:1202.5492.

Kahn, S.M., Leutenegger, M.A., Cottam, J., Rauw, G., Vreux, J.M., den Boggende, A.J.F., Mewe, R., Güdel, M., 2001. High resolution X-ray spectroscopy of zeta Puppis with the XMM-Newton reflection grating spectrometer. A\&A 365, L312L317. doi:10 . 1051/0004-6361:20000093, arXiv: astro-ph/0011026.

Kaper, L., Henrichs, H.F., Nichols, J.S., Telting, J.H., 1999. Long- and short-term variability in O-star winds. II. Quantitative analysis of DAC behaviour. A\&A 344, 231262. arXiv:astro-ph/9812427.

Kramer, R.H., Cohen, D.H., Owocki, S.P., 2003. X-Ray Emission-Line Profile Modeling of O Stars: Fitting a Spherically Symmetric Analytic Wind-Shock Model to the Chandra Spectrum of $\zeta$ Puppis. ApJ 592, 532-538. doi:10.1086/375390, arXiv: astro-ph/0211550.

Krtička, J., 2014. Mass loss in mainsequence B stars. A\&A 564, A70. doi:10.1051/0004-6361/201321980, arXiv: 1401.5511.

Krtička, J., Feldmeier, A., Oskinova, L.M., Kubát, J., Hamann, W.R., 2009. X-ray emission from hydrodynamical simulations in non-LTE wind models. A\&A 508, 841848. doi:10.1051/0004-6361/200912642, arXiv:0910.3430.

Krtička, J., Kubát, J., 2000. Isothermal twocomponent stellar wind of hot stars. A\&A 359, 983-990.

Krtička, J., Kubát, J., 2012. Influence of extreme ultraviolet radiation on the $\mathrm{P} \mathrm{V}$ ionization fraction in hot star winds. MNRAS 427, 84-90. doi:10.1111/j.1365-2966.2012.21895.x, arXiv:1208.1834.

Lamers, H.J.G.L.M., Cassinelli, J.P., 1999. Introduction to Stellar Winds.

Langer, N., 2012. Presupernova Evolution of Massive Single and Binary Stars. ARA\&A 50, 107-164. 
doi:10.1146/annurev-astro-081811-125534, and Analysis of Helium-like Triplet RaarXiv:1206.5443.

Langer, N., Hamann, W.R., Lennon, M., Najarro, F., Pauldrach, A.W.A., Puls, J., 1994. Towards an understanding of very massive stars. A new evolutionary scenario relating $\mathrm{O}$ stars, LBVs and Wolf-Rayet stars. A\&A 290, 819-833.

Lépine, S., Moffat, A.F.J., 1999. Wind Inhomogeneities in Wolf-Rayet Stars. II. Investigation of Emission-Line Profile Variations. ApJ 514, 909-931. doi:10.1086/306958.

Lépine, S., Moffat, A.F.J., 2008. Direct Spectroscopic Observations of Clumping in O-Star Winds. AJ 136, 548553. doi:10.1088/0004-6256/136/2/548, arXiv:0805.1864.

Leutenegger, M.A., Cohen, D.H., Sundqvist, J.O., Owocki, S.P., 2013. Constraints on Porosity and Mass Loss in O-star Winds from the Modeling of X-Ray Emission Line Profile Shapes. ApJ 770, 80. doi:10.1088/0004-637X/770/1/80, arXiv:1305. 5595.

Leutenegger, M.A., Cohen, D.H., Zsargó, J., Martell, E.M., MacArthur, J.P., Owocki, S.P., Gagné, M., Hillier, D.J., 2010. Modeling Broadband X-ray Absorption of Massive Star Winds. ApJ 719, 1767-1774. doi:10.1088/0004-637X/719/2/1767, arXiv: 1007.0783.

Leutenegger, M.A., Paerels, F.B.S., Kahn, S.M., Cohen, D.H., 2006. Measurements tios in the X-Ray Spectra of O-Type Stars. ApJ 650, 1096-1110. doi:10.1086/507147, arXiv:astro-ph/0606370.

Lobel, A., Blomme, R., 2008. Modeling Ultraviolet Wind Line Variability in Massive Hot Stars. ApJ 678, 408-430. doi:10.1086/529129, arXiv:0712.3804.

Lomax, J.R., Nazé, Y., Hoffman, J.L., Russell, C.M.P., De Becker, M., Corcoran, M.F., Davidson, J.W., Neilson, H.R., Owocki, S., Pittard, J.M., Pollock, A.M.T., 2015. V444 Cygni X-ray and polarimetric variability: Radiative and Coriolis forces shape the wind collision region. A\&A 573, A43. doi:10.1051/0004-6361/201424468, arXiv:1410.6117.

Long, K.S., White, R.L., 1980. A survey of soft X-ray emission from hot stars. ApJ 239, L65-L68. doi:10.1086/183293.

Lucy, L.B., 1982. X-ray emission from the winds of hot stars. II. ApJ 255, 286-292. doi:10.1086/159827.

Lucy, L.B., 2012. Coronal winds powered by radiative driving. A\&A 544, A120. doi:10.1051/0004-6361/201118753, arXiv: 1201.0483.

Lucy, L.B., White, R.L., 1980. X-ray emission from the winds of hot stars. ApJ 241, 300-305. doi:10.1086/158342.

Macfarlane, J.J., Cassinelli, J.P., Welsh, B.Y., Vedder, P.W., Vallerga, J.V., Waldron, W.L., 1991. Predicted extreme- 
ultraviolet line emission for nearby mainsequence B stars. ApJ 380, 564-574. doi:10.1086/170614.

Macfarlane, J.J., Cohen, D.H., Wang, P., 1994. X-ray induced ionization in the winds of near-main-sequence $\mathrm{O}$ and $\mathrm{B}$ stars. ApJ 437, 351-360. doi:10.1086/175001.

Marcolino, W.L.F., Bouret, J.C., Martins, F., Hillier, D.J., Lanz, T., Escolano, C., 2009. Analysis of Galactic latetype $\mathrm{O}$ dwarfs: more constraints on the weak wind problem. A\&A 498, 837852. doi:10.1051/0004-6361/200811289, arXiv:0902.1833.

Markova, N., Puls, J., Scuderi, S., Markov, H., 2005. Bright OB stars in the Galaxy. II. Wind variability in $\mathrm{O}$ supergiants as traced by $\mathrm{H} \alpha$. A\&A 440, 11331151. doi:10.1051/0004-6361:20041774, arXiv:astro-ph/0505613.

Martínez-Núñez, S., Torrejón, J.M., Kühnel, M., Kretschmar, P., Stuhlinger, M., RodesRoca, J.J., Fürst, F., Kreykenbohm, I., Martin-Carrillo, A., Pollock, A.M.T., Wilms, J., 2014. The accretion environment in Vela X-1 during a flaring period using XMM-Newton. A\&A 563, A70. doi:10.1051/0004-6361/201322404, arXiv: 1401.2848.

Martins, F., Marcolino, W., Hillier, D.J., Donati, J.F., Bouret, J.C., 2015. Radial dependence of line profile variability in seven O9-B0.5 stars. A\&A 574, A142. doi:10.1051/0004-6361/201423882, arXiv:1409.5057.
Martins, F., Schaerer, D., Hillier, D.J., Meynadier, F., Heydari-Malayeri, M., Walborn, N.R., 2005. On stars with weak winds: the Galactic case. A\&A 441, 735762. doi:10.1051/0004-6361:20052927, arXiv: astro-ph/0507278.

Maryeva, O., Abolmasov, P., 2012. Modelling the optical spectrum of Romano's star. MNRAS 419, 1455-1464. doi:10.1111/j.1365-2966.2011.19802.x, arXiv:1109.0443.

Massa, D., Fullerton, A.W., Sonneborn, G., Hutchings, J.B., 2003. Constraints on the Ionization Balance of Hot-Star Winds from FUSE Observations of $\mathrm{O}$ Stars in the Large Magellanic Cloud. ApJ 586, 996-1018. doi:10.1086/367786, arXiv: astro-ph/0211518.

Massa, D., Oskinova, L., Fullerton, A.W., Prinja, R.K., Bohlender, D.A., Morrison, N.D., Blake, M., Pych, W., 2014. CIR modulation of the X-ray flux from the O7.5 III(n)((f)) star $\xi$ Persei. MNRAS 441, 2173-2180. doi:10.1093/mnras/stu565, arXiv:1403.5601.

Massa, D., Prinja, R.K., 2015. On the Origin of Wind Line Variability in O Stars. ApJ 809, 12. doi:10. 1088/0004-637X/809/1/12, arXiv: 1506.06605.

Mauerhan, J.C., Van Dyk, S.D., Morris, P.W., 2011. Red Eyes on WolfRayet Stars: 60 New Discoveries via Infrared Color Selection. AJ 142, 
40. doi:10.1088/0004-6256/142/2/40, arXiv:1105.5134.

Mewe, R., Raassen, A.J.J., Cassinelli, J.P., van der Hucht, K.A., Miller, N.A., Güdel, M., 2003. High-resolution Xray spectroscopy of tau Scorpii (B0.2V) with XMM-Newton. A\&A 398, 203-211. doi:10.1051/0004-6361:20021577.

Montez, Jr., R., Kastner, J.H., Humphreys, R.M., Turok, R.L., Davidson, K., 2015. Constraints on the Surface Magnetic Fields and Age of a Cool Hypergiant: XMM-Newton X-Ray Observations of VY CMa. ApJ 800, 4. doi:10.1088/0004-637X/800/1/4, arXiv:1412.5120.

Muijres, L.E., Vink, J.S., de Koter, A., Müller, P.E., Langer, N., 2012. Predictions for mass-loss rates and terminal wind velocities of massive O-type stars. A\&A 537, A37. doi:10.1051/0004-6361/201015818, arXiv: 1112.0944.

Mullan, D.J., 1984. Corotating interaction regions in stellar winds. ApJ 283, 303-312. doi:10.1086/162307.

Mullan, D.J., Waldron, W.L., 2006. Winds from OB Stars: A TwoComponent Scenario? ApJ 637, 506-517. doi:10.1086/498253.

Najarro, F., Hanson, M.M., Puls, J., 2011. L-band spectroscopy of Galactic OB-stars. A\&A 535, A32. doi:10.1051/0004-6361/201016003, arXiv:1108.5752.
Nazé, Y., 2009. Hot stars observed by XMMNewton. I. The catalog and the properties of OB stars. A\&A 506, 1055-1064. doi:10.1051/0004-6361/200912659, arXiv:0908.1461.

Nazé, Y., Broos, P.S., Oskinova, L., Townsley, L.K., Cohen, D., Corcoran, M.F., Evans, N.R., Gagné, M., Moffat, A.F.J., Pittard, J.M., Rauw, G., ud-Doula, A., Walborn, N.R., 2011. Global X-ray Properties of the $\mathrm{O}$ and B Stars in Carina. ApJS 194, 7. doi:10.1088/0067-0049/194/1/7, arXiv:1103.0101.

Nazé, Y., Corcoran, M.F., Koenigsberger, G., Moffat, A.F.J., 2007. First Detection of Phase-dependent Colliding Wind X-Ray Emission outside the Milky Way. ApJ 658, L25-L28. doi:10.1086/513510, arXiv: astro-ph/0702403.

Nazé, Y., Oskinova, L.M., Gosset, E., 2013. A Detailed X-Ray Investigation of $\zeta$ Puppis. II. The Variability on Short and Long Timescales. ApJ 763, 143. doi:10.1088/0004-637X/763/2/143, arXiv: 1212.1554.

Nazé, Y., Petit, V., Rinbrand, M., Cohen, D., Owocki, S., ud-Doula, A., Wade, G.A., 2014. X-Ray Emission from Magnetic Massive Stars. ApJS 215, 10. doi:10.1088/0067-0049/215/1/10, arXiv: 1409.1690.

Nazé, Y., Rauw, G., 2008. Highresolution X-ray spectroscopy of $\theta$ Carinae. A\&A 490, 801-806. 
doi:10.1051/0004-6361:200810364, arXiv:0808. 3353.

Nazé, Y., Rauw, G., Hutsemékers, D., 2012. The first X-ray survey of Galactic luminous blue variables. A\&A 538, A47. doi:10.1051/0004-6361/201118040, arXiv:1111.6375.

Nebot Gómez-Morán, A., Motch, C., Pineau, F.X., Carrera, F.J., Pakull, M.W., Riddick, F., 2015. Infrared identification of hard X-ray sources in the Galaxy. MNRAS 452, 884901. doi:10.1093/mnras/stv1020, arXiv: 1506.03083.

Neilson, H.R., Lester, J.B., 2008. On the Enhancement of Mass Loss in Cepheids Due to Radial Pulsation. ApJ 684, 569-587. doi:10.1086/588650, arXiv:0803.4198.

Nichols, J., Huenemoerder, D.P., Corcoran, M.F., Waldron, W., Nazé, Y., Pollock, A.M.T., Moffat, A.F.J., Lauer, J., Shenar, T., Russell, C.M.P., Richardson, N.D., Pablo, H., Evans, N.R., Hamaguchi, K., Gull, T., Hamann, W.R., Oskinova, L., Ignace, R., Hoffman, J.L., Hole, K.T., Lomax, J.R., 2015. A Coordinated XRay and Optical Campaign of the Nearest Massive Eclipsing Binary, $\delta$ Orionis Aa. II. X-Ray Variability. ApJ 809, 133. doi:10.1088/0004-637X/809/2/133, arXiv: 1507.04972.

Oskinova, L., 2006. Quantitative Xray Spectroscopy of Massive Stars, in:
High Resolution X-ray Spectroscopy: towards XEUS and Con-X, pp. 27-32. arXiv: astro-ph/0605560.

Oskinova, L.M., 2005. Evolution of X-ray emission from young massive star clusters. MNRAS 361, 679-694. doi:10.1111/j.1365-2966.2005.09229.x, arXiv:astro-ph/0505512.

Oskinova, L.M., Clarke, D., Pollock, A.M.T., 2001. Rotationally modulated X-ray emission from the single $\mathrm{O}$ star $\zeta$ Ophiuchi. A\&A 378, L21-L24. doi:10.1051/0004-6361:20011222.

Oskinova, L.M., Feldmeier, A., Hamann, W.R., 2004. X-ray emission lines from inhomogeneous stellar winds. A\&A 422, 675691. doi:10.1051/0004-6361:20047187, arXiv: astro-ph/0403707.

Oskinova, L.M., Feldmeier, A., Hamann, W.R., 2006. High-resolution Xray spectroscopy of bright O-type stars. MNRAS 372, 313-326. doi:10.1111/j.1365-2966.2006.10858.x, arXiv: astro-ph/0603286.

Oskinova, L.M., Gayley, K.G., Hamann, W.R., Huenemoerder, D.P., Ignace, R., Pollock, A.M.T., 2012. High-resolution XRay Spectroscopy Reveals the Special Nature of Wolf-Rayet Star Winds. ApJ 747, L25. doi:10. 1088/2041-8205/747/2/L25, arXiv:1202.1525.

Oskinova, L.M., Hamann, W.R., 2008. Puzzling X-rays from the new colliding wind binary Wolf-Rayet 65 
(WC9d). MNRAS 390, L78-L82. doi:10.1111/j.1745-3933.2008.00542.x, arXiv:0809.2782.

Oskinova, L.M., Hamann, W.R., Cassinelli, J.P., Brown, J.C., Todt, H., 2011a. X-ray emission from massive stars with magnetic fields. Astronomische Nachrichten 332, 988-993. doi:10.1002/asna.201111602, arXiv:1110.5345.

Oskinova, L.M., Hamann, W.R., Feldmeier, A., 2007. Neglecting the porosity of hot-star winds can lead to underestimating mass-loss rates. A\&A 476, 13311340. doi:10.1051/0004-6361:20066377, arXiv:0704.2390.

Oskinova, L.M., Hamann, W.R., Feldmeier, A., Ignace, R., Chu, Y.H., 2009. Discovery of X-Ray Emission from the Wolf-Rayet Star WR 142 of Oxygen Subtype. ApJ 693, L44L48. doi:10.1088/0004-637X/693/1/L44, arXiv:0901. 4553.

Oskinova, L.M., Ignace, R., Hamann, W.R., Pollock, A.M.T., Brown, J.C., 2003. The conspicuous absence of X-ray emission from carbon-enriched Wolf-Rayet stars. A\&A 402, 755765. doi:10.1051/0004-6361:20030300, arXiv: astro-ph/0303025.

Oskinova, L.M., Nazé, Y., Todt, H., Huenemoerder, D.P., Ignace, R., Hubrig, S., Hamann, W.R., 2014. Discovery of X-ray pulsations from a massive star. Nature Communications
5, 4024. doi:10.1038/ncomms5024, arXiv: 1406.0845.

Oskinova, L.M., Todt, H., Huenemoerder, D.P., Hubrig, S., Ignace, R., Hamann, W.R., Balona, L., 2015. On X-ray pulsations in $\beta$ Cephei-type variables. A\&A 577, A32. doi:10.1051/0004-6361/201525908, arXiv: 1503.05749.

Oskinova, L.M., Todt, H., Ignace, R., Brown, J.C., Cassinelli, J.P., Hamann, W.R., 2011b. Early magnetic Btype stars: X-ray emission and wind properties. MNRAS 416, 1456-1474. doi:10.1111/j.1365-2966.2011.19143.x, arXiv:1106.0508.

Owocki, S.P., 2008. Dynamical simulation of the "velocity-porosity" reduction in observed strength of stellar wind lines, in: Hamann, W.R., Feldmeier, A., Oskinova, L.M. (Eds.), Clumping in Hot-Star Winds: proceedings of an international workshop held in Potsdam, Germany, 18. - 22. June 2007, Universitätsverlag Potsdam., p. 121124.

Owocki, S.P., Castor, J.I., Rybicki, G.B., 1988. Time-dependent models of radiatively driven stellar winds. I - Nonlinear evolution of instabilities for a pure absorption model. ApJ 335, 914-930. doi:10.1086/166977.

Owocki, S.P., Cohen, D.H., 1999. A Simple Scaling Analysis of X-Ray Emission and Absorption in Hot-Star Winds. ApJ 520, 833-840. doi:10.1086/307500, arXiv: astro-ph/9901250. 
Owocki, S.P., Cohen, D.H., 2001. X-Ray Line Profiles from Parameterized Emission within an Accelerating Stellar Wind. ApJ 559, 1108-1116. doi:10.1086/322413, arXiv: astro-ph/0101294.

Owocki, S.P., Cohen, D.H., 2006. The Effect of Porosity on X-Ray EmissionLine Profiles from Hot-Star Winds. ApJ 648, 565-571. doi:10.1086/505698, arXiv: astro-ph/0602054.

Owocki, S.P., Gayley, K.G., Shaviv, N.J., 2004. A Porosity-Length Formalism for Photon-Tiring-limited Mass Loss from Stars above the Eddington Limit. ApJ 616, 525-541. doi:10.1086/424910, arXiv: astro-ph/0409573.

Owocki, S.P., Puls, J., 2002. Ion Runaway Instability in Low-Density, Linedriven Stellar Winds. ApJ 568, 965-978. doi:10.1086/339037.

Owocki, S.P., Sundqvist, J.O., Cohen, D.H., Gayley, K.G., 2013. Thin-shell mixing in radiative wind-shocks and the $L_{x} \propto L_{b o l}$ scaling of O-star X-rays. MNRAS 429, 3379-3389. doi:10.1093/mnras/sts599, arXiv: 1212.4235 .

Pallavicini, R., 1989. X-ray emission from stellar coronae. A\&A Rev. 1, 177-207. doi:10.1007/BF00872715.

Pallavicini, R., Golub, L., Rosner, R., Vaiana, G.S., Ayres, T., Linsky, J.L., 1981. Relations among stellar X-ray emission observed from Einstein, stellar rotation and bolometric luminosity. ApJ 248, 279-290. doi:10.1086/159152.

Parkin, E.R., Pittard, J.M., Corcoran, M.F., Hamaguchi, K., Stevens, I.R., 2009. 3D modelling of the colliding winds in $\eta$ Carinae - evidence for radiative inhibition. MNRAS 394, 1758-1774. doi:10.1111/j.1365-2966.2009.14475.x, arXiv:0901.0862.

Petit, V., Massa, D.L., Marcolino, W.L.F., Wade, G.A., Ignace, R., Mimes Collaboration, 2011. Discovery of the first $\tau$ Sco analogues: HD 66665 and HD 63425. MNRAS 412, L45-L49. doi:10.1111/j.1745-3933.2010.01002.x, arXiv: 1012.4445.

Pillitteri, I., Wolk, S.J., Goodman, A., Sciortino, S., 2014. Smooth X-ray variability from $\rho$ Ophiuchi A+B. A strongly magnetized primary B2 star? A\&A 567, L4. doi:10.1051/0004-6361/201424243, arXiv: 1406.5049 .

Pollock, A.M.T., 1987. The Einstein view of the Wolf-Rayet stars. ApJ 320, 283-295. doi:10. 1086/165539.

Pollock, A.M.T., 2007. A new paradigm for the X-ray emission of $\mathrm{O}$ stars from XMM-Newton observations of the O9.7 supergiant $\zeta$ Orionis. A\&A 463, 11111123. doi:10.1051/0004-6361:20053838, arXiv: astro-ph/0612500.

Pollock, A.M.T., Corcoran, M.F., Stevens, I.R., Williams, P.M., 2005. Bulk Velocities, Chemical Composition, and Ioniza- 
tion Structure of the X-Ray Shocks in WR 140 near Periastron as Revealed by the Chandra Gratings. ApJ 629, 482-498. doi:10.1086/431193.

Pollock, A.M.T., Haberl, F., Corcoran, M.F., 1995. The ROSAT PSPC survey of the Wolf-Rayet stars, in: van der Hucht, K.A., Williams, P.M. (Eds.), Wolf-Rayet Stars: Binaries; Colliding Winds; Evolution, Kluwer Academic Publishers; Dordrecht; p. 512-51.

Porquet, D., Mewe, R., Dubau, J., Raassen, A.J.J., Kaastra, J.S., 2001. Line ratios for helium-like ions: Applications to collisiondominated plasmas. A\&A 376, 11131122. doi:10.1051/0004-6361:20010959, arXiv:astro-ph/0107329.

Portegies Zwart, S.F., van den Heuvel, E.P.J., 2016. Was the nineteenth century giant eruption of Eta Carinae a merger event in a triple system? MNRAS 456, 3401-3412. doi:10.1093/mnras/stv2787, arXiv: 1511.06889.

Prinja, R.K., 1989. Ultraviolet observations of stellar winds in Be and 'normal' B nonsupergiant stars. MNRAS 241, 721-752.

Prinja, R.K., Massa, D.L., 2010. Signature of wide-spread clumping in $\mathrm{B}$ supergiant winds. A\&A 521, L55. doi:10.1051/0004-6361/201015252, arXiv:1007. 2744.

Puebla, R.E., Hillier, D.J., Zsargó, J., Cohen, D.H., Leutenegger, M.A., 2016.
X-ray, UV and optical analysis of supergiants: $\epsilon$ Ori. MNRAS 456, 29072936. doi:10.1093/mnras/stv2783, arXiv: 1511.09365.

Puls, J., Markova, N., Scuderi, S., Stanghellini, C., Taranova, O.G., Burnley, A.W., Howarth, I.D., 2006. Bright OB stars in the Galaxy. III. Constraints on the radial stratification of the clumping factor in hot star winds from a combined $\mathrm{H}, \mathrm{IR}$ and radio analysis. A\&A 454, 625651. doi:10.1051/0004-6361:20065073, arXiv:astro-ph/0604372.

Raassen, A.J.J., Cassinelli, J.P., Miller, N.A., Mewe, R., Tepedelenlioğlu, E., 2005. XMM-Newton observations of $\beta$ Centauri (B1 III): The temperature structure in the hot plasma and the photospherewind connection. A\&A 437, 599-609. doi:10 . 1051/0004-6361:20052650.

Ramiaramanantsoa, T., Moffat, A.F.J., Chené, A.N., Richardson, N.D., Henrichs, H.F., Desforges, S., Antoci, V., Rowe, J.F., Matthews, J.M., Kuschnig, R., Weiss, W.W., Sasselov, D., Rucinski, S.M., Guenther, D.B., 2014. MOST detects corotating bright spots on the mid-O-type giant $\xi$ Persei. MNRAS 441, 910-917. doi:10.1093/mnras/stu619, arXiv: 1403.7843.

Rauw, G., Hervé, A., Nazé, Y., GonzálezPérez, J.N., Hempelmann, A., Mittag, M., Schmitt, J.H.M.M., Schröder, K.P., Gosset, E., Eenens, P., UuhSonda, J.M., 2015a. Simultaneous X- 
ray and optical spectroscopy of the Oef supergiant $\lambda$ Cephei. A\&A 580, A59. doi:10.1051/0004-6361/201526057, arXiv: 1505.07714.

Rauw, G., Nazé, Y., Wright, N.J., Drake, J.J., Guarcello, M.G., Prinja, R.K., Peck, L.W., Albacete Colombo, J.F., Herrero, A., Kobulnicky, H.A., Sciortino, S., Vink, J.S., 2015b. X-Ray Emission from Massive Stars in Cyg OB2. ApJS 221, 1. doi:10.1088/0067-0049/221/1/1, arXiv: 1401.8098.

Runacres, M.C., Owocki, S.P., 2002. The outer evolution of instabilitygenerated structure in radiatively driven stellar winds. A\&A 381, 1015-1025. doi:10.1051/0004-6361:20011526.

Sana, H., Rauw, G., Nazé, Y., Gosset, E., Vreux, J.M., 2006. An XMMNewton view of the young open cluster NGC 6231 - II. The OB star population. MNRAS 372, 661-678. doi:10.1111/j.1365-2966.2006.10847.x, arXiv: astro-ph/0607486.

Sander, A., Hamann, W.R., Todt, H., 2012. The Galactic WC stars. Stellar parameters from spectral analyses indicate a new evolutionary sequence. A\&A 540, A144. doi:10.1051/0004-6361/201117830, arXiv:1201.6354.

Schnerr, R.S., Henrichs, H.F., Neiner, C., Verdugo, E., de Jong, J., Geers, V.C., Wiersema, K., van Dalen, B., Tijani, A., Plaggenborg, B., Rygl,
K.L.J., 2008. Magnetic field measurements and wind-line variability of OB-type stars. A\&A 483, 857-867. doi:10.1051/0004-6361:20077740, arXiv:1008.4260.

Schnerr, R.S., Henrichs, H.F., Oudmaijer, R.D., Telting, J.H., 2006. On the $\mathrm{H} \alpha$ emission from the ¡ASTROBJ ${ }_{i} \beta \quad$ Cephei $/$ ASTROBJ system. A\&A 459, L21-L24. doi:10.1051/0004-6361:20066392, arXiv: arXiv: astro-ph/0610198.

Schulz, N.S., Canizares, C.R., Lee, J.C., Sako, M., 2002. The Ionized Stellar Wind in Vela X-1 during Eclipse. ApJ 564, L21-L25. doi:10.1086/338862, arXiv: astro-ph/0111489.

Seward, F.D., Forman, W.R., Giacconi, R., Griffiths, R.E., Harnden, Jr., F.R., Jones, C., Pye, J.P., 1979. X-rays from Eta Carinae and the surrounding nebula. ApJ 234, L55-L58. doi:10.1086/183108.

Shenar, T., Oskinova, L., Hamann, W.R., Corcoran, M.F., Moffat, A.F.J., Pablo, H., Richardson, N.D., Waldron, W.L., Huenemoerder, D.P., Maíz Apellániz, J., Nichols, J.S., Todt, H., Nazé, Y., Hoffman, J.L., Pollock, A.M.T., Negueruela, I., 2015. A Coordinated X-Ray and Optical Campaign of the Nearest Massive Eclipsing Binary, $\delta$ Orionis Aa. IV. A Multiwavelength, Non-LTE Spectroscopic Analysis. ApJ 809, 135. doi:10.1088/0004-637X/809/2/135, arXiv: 1503.03476. 
Skinner, S.L., Sokal, K.R., Cohen, D.H., Gagné, M., Owocki, S.P., Townsend, R.D., 2008. High-Resolution Chandra $\mathrm{X}$-Ray Imaging and Spectroscopy of the $\sigma$ Orionis Cluster. ApJ 683, 796-812. doi:10.1086/589917, arXiv:0805.0714.

Skinner, S.L., Zhekov, S.A., Güdel, M., Schmutz, W., 2002. XMM-Newton and Very Large Array Observations of the Variable Wolf-Rayet Star EZ Canis Majoris: Evidence for a Close Companion? ApJ 579, 764-773. doi:10.1086/342841, arXiv:astro-ph/0207171.

Skinner, S.L., Zhekov, S.A., Güdel, M., Schmutz, W., Sokal, K.R., 2010. Xray Emission from Nitrogen-Type Wolf-Rayet Stars. AJ 139, 825-838. doi:10.1088/0004-6256/139/3/825, arXiv:0912.1326.

Skinner, S.L., Zhekov, S.A., Güdel, M., Schmutz, W., Sokal, K.R., 2012. New XRay Detections of WNL Stars. AJ 143, 116. doi:10.1088/0004-6256/143/5/116, arXiv: 1203.5098.

Sokal, K.R., Skinner, S.L., Zhekov, S.A., Güdel, M., Schmutz, W., 2010. Chandra Detects the Rare Oxygen-type WolfRayet Star WR 142 and OB Stars in Berkeley 87. ApJ 715, 1327-1337. doi:10.1088/0004-637X/715/2/1327, arXiv: 1004.0462.

Springmann, U.W.E., Pauldrach, A.W.A., 1992. Radiation-driven winds of hot luminous stars. XI - Frictional heating in a multicomponent stellar wind plasma and decoupling of radiatively accelerated ions. A\&A 262, 515-522.

Stelzer, B., Flaccomio, E., Montmerle, T., Micela, G., Sciortino, S., Favata, F., Preibisch, T., Feigelson, E.D., 2005. X-Ray Emission from Early-Type Stars in the Orion Nebula Cluster. ApJS 160, 557-581. doi:10.1086/432375, arXiv: astro-ph/0505503.

Stewart, G.C., Fabian, A.C., 1981. The influence of mass loss on the observed X-ray spectra of early-type stars. MNRAS 197, 713-720.

Sugawara, Y., Tsuboi, Y., Maeda, Y., 2008. Redshifted emission lines and radiative recombination continuum from the WolfRayet binary $\theta$ Muscae: evidence for a triplet system? A\&A 490, 259264. doi:10.1051/0004-6361:20079302, arXiv:0810.1208.

Sundqvist, J.O., Owocki, S.P., Cohen, D.H., Leutenegger, M.A., Townsend, R.H.D., 2012. A generalized porosity formalism for isotropic and anisotropic effective opacity and its effects on $\mathrm{X}$-ray line attenuation in clumped O star winds. MNRAS 420, 1553-1561. doi:10.1111/j.1365-2966.2011.20141.x, arXiv:1111.1762.

Sundqvist, J.O., Puls, J., Feldmeier, A., 2010. Mass loss from inhomogeneous hot star winds. I. Resonance line formation in 2D models. A\&A 510, 
A11. doi:10.1051/0004-6361/200912842, arXiv:0911.3361.

Torrejón, J.M., Schulz, N.S., Nowak, M.A., Oskinova, L., Rodes-Roca, J.J., Shenar, T., Wilms, J., 2015. On the Radial Onset of Clumping in the Wind of the B0I Massive Star QV Nor. ApJ 810, 102. doi:10.1088/0004-637X/810/2/102.

Tramper, F., Straal, S.M., Sanyal, D., Sana, H., de Koter, A., Gräfener, G., Langer, N., Vink, J.S., de Mink, S.E., Kaper, L., 2015. Massive stars on the verge of exploding: the properties of oxygen sequence Wolf-Rayet stars. A\&A 581, A110. doi:10.1051/0004-6361/201425390, arXiv: 1507.00839.

Tuthill, P.G., Monnier, J.D., Danchi, W.C., 1999. A dusty pinwheel nebula around the massive star WR104. Nature 398, 487-489. doi:10.1038/19033, arXiv:astro-ph/9904092.

Tutukov, A.V., Fedorova, A.V., 2010. Possible scenarios for the formation of $\mathrm{Ap} / \mathrm{Bp}$ stars. Astronomy Reports 54, 156-162. doi:10.1134/S1063772910020083.

Tylenda, R., Soker, N., 2006. Eruptions of the V838 Mon type: stellar merger versus nuclear outburst models. A\&A 451, 223236. doi:10.1051/0004-6361:20054201, arXiv: astro-ph/0509379.

Šurlan, B., Hamann, W.R., Aret, A., Kubát, J., Oskinova, L.M., Torres, A.F., 2013.
Macroclumping as solution of the discrepancy between $\mathrm{H} \alpha$ and $\mathrm{P}$ v mass loss diagnostics for O-type stars. A\&A 559, A130. doi:10.1051/0004-6361/201322390, arXiv: 1310.0449 .

Šurlan, B., Hamann, W.R., Kubát, J., Oskinova, L.M., Feldmeier, A., 2012. Threedimensional radiative transfer in clumped hot star winds. I. Influence of clumping on the resonance line formation. A\&A 541, A37. doi:10.1051/0004-6361/201118590, arXiv: 1202.4787.

van der Meer, A., Kaper, L., di Salvo, T., Méndez, M., van der Klis, M., Barr, P., Trams, N.R., 2005. XMM-Newton X-ray spectroscopy of the high-mass X-ray binary $4 \mathrm{U}$ 1700-37 at low flux. A\&A 432, 9991012. doi:10.1051/0004-6361:20041288, arXiv: astro-ph/0412021.

Verner, D.A., Yakovlev, D.G., 1995. Analytic FITS for partial photoionization cross sections. A\&AS 109, 125-133.

Vink, J.S., de Koter, A., Lamers, H.J.G.L.M., 2001. Mass-loss predictions for $\mathrm{O}$ and $\mathrm{B}$ stars as a function of metallicity. A\&A 369, 574-588. doi:10.1051/0004-6361:20010127, arXiv: astro-ph/0101509.

Vishniac, E.T., 1994. Nonlinear instabilities in shock-bounded slabs. ApJ 428, 186-208. doi:10.1086/174231, arXiv:astro-ph/9306025.

Walborn, N.R., Nichols, J.S., Waldron, W.L., 2009. The Correlation Between X- 
Ray Line Ionization and Optical Spectral Types of the OB Stars. ApJ 703, 633641. doi:10.1088/0004-637X/703/1/633, arXiv: 0907.4738.

Waldron, W.L., 1984. Recombination stellar wind model for the coronae of early-type stars. ApJ 282, 256-266. doi:10.1086/162198.

Waldron, W.L., Cassinelli, J.P., 2001. Chandra Discovers a Very High Density XRay Plasma on the O Star $\zeta$ Orionis. ApJ 548, L45-L48. doi:10.1086/318926, arXiv:astro-ph/0012190.

Waldron, W.L., Cassinelli, J.P., 2007. An Extensive Collection of Stellar Wind XRay Source Region Emission Line Parameters, Temperatures, Velocities, and Their Radial Distributions as Obtained from Chandra Observations of 17 OB Stars. ApJ 668, 456-480. doi:10.1086/520919, arXiv:0707.0024.

Waldron, W.L., Cassinelli, J.P., 2009. Highly Accelerated Diamagnetic Plasmoids: A New X-Ray Production Mechanism for OB Stellar Winds. ApJ 692, L76L79. doi:10 . 1088/0004-637X/692/2/L76, arXiv:0901.1405.

Waldron, W.L., Cassinelli, J.P., 2010. The Importance of XUV Radiation as a Solution to the $\mathrm{P} V$ Mass Loss Rate Discrepancy in O Stars. ApJ 711, L30 L34. doi:10.1088/2041-8205/711/1/L30, arXiv: 1001.4512.
Wessolowski, U., 1996. X-ray emission from (putatively) single WN-type W-R stars., in: Zimmermann, H.U., Trümper, J., Yorke, H. (Eds.), Roentgenstrahlung from the Universe, Proceedings to the Wuerzburg Conference, published by the MPI für Extraterrestrische Physik, Wurzburg, Germany p. 75-76.

Williams, P.M., van der Hucht, K.A., Pollock, A.M.T., Florkowski, D.R., van der Woerd, H., Wamsteker, W.M., 1990. Multi-frequency variations of the WolfRayet system HD 193793. I - Infrared, Xray and radio observations. MNRAS 243, 662-684.

Wrigge, M., Wendker, H.J., Wisotzki, L., 1994. X-ray emission from wind blown interstellar bubbles I. ROSAT observations of NGC 6888. A\&A 286, 219-230.

Zhekov, S.A., 2012. X-rays from colliding stellar winds: the case of close Wolf-Rayet+O binary systems. MNRAS 422, 1332-1342. doi:10.1111/j.1365-2966.2012.20706.x, arXiv: 1202.1386.

Zhekov, S.A., Palla, F., 2007. X-rays from massive OB stars: thermal emission from radiative shocks. MNRAS 382, 1124-1132. doi:10.1111/j.1365-2966.2007.12286.x, arXiv:0708.0085.

Zsargó, J., Hillier, D.J., Bouret, J.C., Lanz, T., Leutenegger, M.A., Cohen, D.H., 2008. On the Importance of the Interclump Medium for Superionization: O VI Formation in the Wind of $\zeta$ Puppis. ApJ 
685, L149-L152. doi:10.1086/592568, arXiv:0808. 2233. 